\title{
String field theory vertex from integrability
}

\author{
Zoltan Bajnok $^{a}$ and Romuald A. Janik ${ }^{b}$ \\ ${ }^{a}$ MTA Lendület Holographic QFT Group, Wigner Research Centre, \\ H-1525 Budapest 114, P.O.B. 49, Hungary \\ ${ }^{b}$ Institute of Physics, Jagiellonian University, \\ ul. Eojasiewicza 11, 30-348 Kraków, Poland \\ E-mail: bajnok.zoltan@wigner.mta.hu, romuald@th.if.uj.edu.pl
}

ABSTRACT: We propose a framework for computing the (light cone) string field theory vertex in the case when the string worldsheet QFT is a generic integrable theory. The prime example and ultimate goal would be the $A d S_{5} \times S^{5}$ superstring theory cubic string vertex and the chief application will be to use this framework as a formulation for $\mathcal{N}=4 \mathrm{SYM}$ theory OPE coefficients valid at any coupling up to wrapping corrections. In this paper we propose integrability axioms for the vertex, illustrate them on the example of the pp-wave string field theory and also uncover similar structures in weak coupling computations of OPE coefficients.

Keywords: AdS-CFT Correspondence, Integrable Field Theories, String Field Theory

ARXIV EPRINT: 1501.04533 


\section{Contents}

1 Introduction 1

2 Insight from the spectral problem and form factors 4

3 The pp-wave light cone string field theory vertex $\quad 8$

4 The decompactified string vertex and the SFT axioms 13

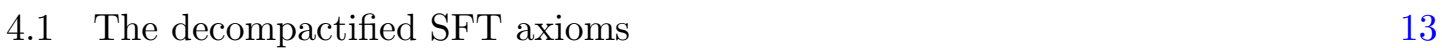

5 The free massive boson example (or the pp-wave SFT vertex) 18

$\begin{array}{lll}5.1 \text { A review of LSNS formulas } & 18\end{array}$

5.2 The decompactification limit of the LSNS formulas and their analyticity $\begin{array}{ll}\text { properties } & 20\end{array}$

5.3 Asymptotic limit 22

5.4 Reconstruction of $\tilde{\Gamma}_{\mu}(\theta)$ from the SFT axioms 24

6 Axioms for the nondiagonal case $\quad 25$

$\begin{array}{lll}7 & \text { The program for the finite volume string vertex } & 29\end{array}$

$\begin{array}{ll}\text { 7.1 The program for the simplest plane wave SFT vertex } & 31\end{array}$

8 Weak coupling cross-checks with OPE coefficients 32

8.1 The $s u(1 \mid 1)$ sector $\quad 33$

8.2 The $s u(2)$ sector 34

9 Conclusions $\quad 35$

$\begin{array}{ll}\text { A The decompactified vertex formulation and solution } & 37\end{array}$

B Properties of the $\tilde{\Gamma}_{\mu}(\theta)$ functions $\quad 41$

C Details on the $s u(1 \mid 1)$ and $s u(2)$ OPE coefficients 42

$\begin{array}{lll}\text { C.1 The } s u(1 \mid 1) \text { sector } & 42\end{array}$

$\begin{array}{lll}\text { C.2 The } s u(2) \text { sector } & 44\end{array}$

\section{Introduction}

The integrability properties of string theory in $A d S_{5} \times S^{5}$ background [1] together with the AdS/CFT correspondence [2] allows for obtaining exact results for various observables in $\mathcal{N}=4$ Super-Yang-Mills (SYM) theory for any value of the gauge theory coupling in the planar, large $N_{c}$ limit. Currently this program is very well developed for the spectral 
problem, namely for the determination of the scaling dimensions of all local operators [3][8]. For other observables we have currently only partial results like various strong and weak coupling expansions or exact answers but restricted to some particular concrete observables like generalized cusp Wilson loops, circular loops or for some ingredients of scattering amplitudes.

A class of observables for which it would be crucial to obtain a similar level of understanding as for the scaling dimensions are the OPE coefficients or, equivalently, the 3-point correlation functions of local operators. Namely, these quantities provide the remaining fundamental data for any conformal field theory (CFT). Indeed, higher point functions do not carry any independent dynamical content and can be reduced to scaling dimensions, OPE coefficients and conformal blocks determined by conformal symmetry alone.

On the string side of the AdS/CFT correspondence these quantities are also interesting for their own sake, namely the AdS/CFT string diagram corresponding to a 3-point function can be interpreted as a three string interaction. In fact, the first wave of interest in OPE coefficients of (unprotected) operators in $\mathcal{N}=4$ SYM theory [9]-[12] came from the proposed link with the 3-string string field theory vertex in the pp-wave [13] string field theory (SFT) [14]-[19]. The SFT vertex is also interesting as it is related to the first $1 / N_{c}$ corrections to the string hamiltonian/scaling dimensions, too.

Unfortunately, there is practically no information on generalizing the pp-wave SFT to the full $A d S_{5} \times S^{5}$ case. This is not an issue of technical or calculational complexity but rather a more fundamental one. A unique feature of the pp-wave geometry is that, although it is curved, the worldsheet quantum field theory of the string in an appropriate light cone gauge reduces to free massive bosons and fermions [20], thus allowing for the use of mode expansions in implementing continuity conditions for the SFT (light cone) vertex [14] similarly as for the flat space SFT vertex [21]. For an interacting worldsheet QFT, as is the case for the full $A d S_{5} \times S^{5}$ geometry, we do not have any techniques so far for finding the SFT vertex.

Thus the main goal of the present paper is to provide a new formulation for the problem of determining the (light cone) SFT vertex in the case when the worldsheet theory is a generic integrable QFT, which includes as a key special case the $A d S_{5} \times S^{5}$ background. We propose an integrable bootstrap formulation of the SFT vertex, namely a set of coupled functional equations for the SFT amplitudes understood as the value of the vertex with specific string excited states on each of the three legs. The dependence on the concrete background/worldsheet QFT enters through the appearance of the S-matrix in the SFT vertex axioms. This formulation should be valid up to exponential 'wrapping corrections'.

The bootstrap approach for obtaining various physical quantities in two dimensional integrable quantum field theories has already a long and successful history. Basically it amounts to implementing very general functional and analyticity properties of the various observables and using in addition key properties of integrability like factorized scattering etc.

Initially, the bootstrap program was developed for determining the scattering amplitudes (and at the same time the particle content, hence the name bootstrap) for a theory on a two-dimensional plane [22]-[24]. The result is the explicit knowledge of the 2-particle scattering S-matrix and the mass spectrum of the theory, e.g. the masses of bound states 


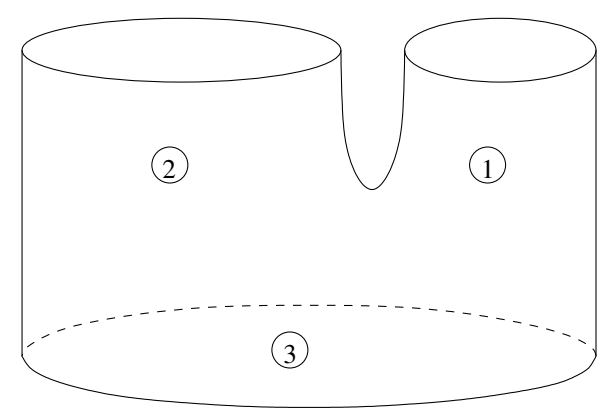

Figure 1. The geometry of the worldsheet for the cubic light cone string field theory vertex.

in terms of the masses of the fundamental particles. Subsequently this information was used to obtain the spectrum of such a theory on a cylinder of finite size [25, 26].

Since then, the bootstrap program was extended to cover theories with integrable boundary conditions [27], providing exact formulas for reflection factors; as well as for theories with integrable defects [28].

A whole new field of research started when bootstrap was applied to more fine-grained, and in a certain sense off-shell observables such as form factors [29]-[31]. Here, in contrast to ordinary scattering amplitudes the number of incoming and outgoing particles does not need to be balanced. All the above developments appeared within the context of ordinary relativistic integrable quantum field theories and reflected the main questions of interest in such contexts.

One of the most intriguing features of the AdS/CFT correspondence is that it provides a mapping between observables in a 4-dimensional gauge theory and in the 2dimensional string worldsheet quantum field theory. As such, some natural questions in the 4-dimensional gauge theory suggest completely novel problems/geometrical configurations in the dual 2-dimensional integrable QFT, which were never investigated hitherto by the relativistic integrable QFT community.

Some prime examples of such problems involve, on the classical level, strong coupling scattering amplitudes (equivalently null polygonal Wilson loops) [32], classical solutions with the topology of a thrice-punctured sphere relevant for the OPE coefficients of (classical) operators at strong coupling [33]-[36].

The first application of bootstrap ideas in such a novel geometrical context was the very interesting work [37], which provided bootstrap equations for (excited) pentagonal scattering amplitudes relevant for general multigluon scattering amplitudes in $\mathcal{N}=4 \mathrm{SYM}$.

The goal of this work is to apply the bootstrap methodology to the classic string pants diagram (see figure 1) relevant for the (light cone) SFT vertex.

In the following section, which is still a continuation of the introduction, we provide an explanation of the main ideas and motivations behind our approach and then give an outline of the remaining parts of the paper. 


\section{Insight from the spectral problem and form factors}

The spectral problem for an integrable quantum field theory is defined as finding the energy levels of the theory defined on a cylinder of arbitrary size.

As a first step in solving this problem, one passes to the same theory but defined in infinite volume - on the whole two-dimensional plane. There we have well defined asymptotic states so we can consider the S-matrix. What is crucial, however, is that only in this setting we have at our disposal analyticity properties of the S-matrix, especially crossing. Thus one first solves the theory in infinite volume by implementing the symmetries of the problem, solving the Yang-Baxter equation together with unitarity and crossing, and determining any remaining possible CDD factors. At this stage one obtains the exact analytical form of the S-matrix. This procedure is commonly called the S-matrix bootstrap.

In the second step, one considers the same theory defined on a large cylinder of circumference $L$. A multiparticle state on the cylinder can be considered just as a quantum mechanical multiparticle state parametrized by the particles momenta $\left\{p_{i}\right\}$. These momenta are quantized by the Asymptotic Bethe Ansatz quantization condition ${ }^{1}$

$$
e^{i \Phi_{k}\left(\left\{p_{i}\right\}\right)} \equiv e^{i p_{k} L} \prod_{i \neq k} S\left(p_{k}, p_{i}\right)=1
$$

which essentially amounts to the single valuedness condition for the wave function. The energy of the relevant state is then given by the sum of the particles' energies

$$
E=\sum_{k=1}^{N} E\left(p_{k}\right) .
$$

As we decrease the size of the cylinder, quantum-field-theoretical virtual effects become important (so-called wrapping corrections) with the leading terms being described by (generalized) Lüscher corrections [38, 39], again in terms of infinite volume data. These corrections give additional terms of order $e^{-m L}$ in the energy formula (2.2) and quantization conditions (2.1). Subsequent multiple wrapping terms are much more involved (although progress has recently been made [40]) but surprisingly the whole infinite set of wrapping corrections can be effectively resummed through the so-called Thermodynamic Bethe Ansatz, which provides the exact spectrum for any size of the cylinder. In the nondiagonal case, this last step is, however, quite involved (this is especially true in the $A d S_{5} \times S^{5}$ setup [3]-[8]).

In the above description we would like to emphasize two points. Firstly, the neccessity of having an infinite volume description in order to formulate functional equations for the S-matrix. Here the existence of crossing invariance is of particular importance. Secondly, the simplicity of the finite volume answer as long as we neglect the exponential wrapping corrections $\sim e^{-m L}$. The obtained answer is valid for any value of the coupling in the integrable QFT. Ultimately we would like to have a similar framework for the OPE coefficients.

A suggestion has been made for the use of form factors in this context [41]-[44].

\footnotetext{
${ }^{1}$ With appropriate nested Bethe Ansatz structure in case of nondiagonal S-matrices.
} 
Form factors are expectation values of a local operator on the worldsheet sandwiched between multiparticle in and out states.

$$
{ }_{\text {out }}\left\langle\theta_{1}^{\prime}, \ldots, \theta_{m}^{\prime}|\mathcal{O}(0)| \theta_{1}, \ldots, \theta_{n}\right\rangle_{\text {in }}
$$

In infinite volume one may use crossing to put all particles into the in state

$$
\left\langle 0|\mathcal{O}(0)| \theta_{1}, \ldots, \theta_{n}\right\rangle \equiv F_{n}\left(\theta_{1}, \ldots, \theta_{n}\right)
$$

and formulate functional equations for these quantities. Assuming for simplicity a theory with just one species of particles and no bound states, the equations take a very transparent form:

$$
\begin{aligned}
F_{n}\left(\theta_{1}, \ldots, \theta_{i}, \theta_{i+1}, \ldots, \theta_{n}\right) & =F_{n}\left(\theta_{1}, \ldots, \theta_{i+1}, \theta_{i}, \ldots, \theta_{n}\right) S\left(\theta_{i}, \theta_{i+1}\right) \\
F_{n}\left(\theta_{1}+2 \pi i, \theta_{2}, \ldots, \theta_{n}\right) & =F_{n}\left(\theta_{2}, \ldots, \theta_{n}, \theta_{1}\right) \\
-i \operatorname{Res}_{\theta^{\prime}=\theta} F_{n+2}\left(\theta^{\prime}+i \pi, \theta, \theta_{1}, \ldots, \theta_{n}\right) & =\left(1-\prod_{i=1}^{n} S\left(\theta, \theta_{i}\right)\right) F_{n}\left(\theta_{1}, \ldots, \theta_{n}\right) .
\end{aligned}
$$

Equation (2.5) can be understood as a simple consequence of the commutation relation between Zamolodchikov-Faddeev creation operators. Equation (2.6) is very important as it involves in a crucial way crossing properties. Last particle with rapidity $\theta_{1}$ gets crossed up to the out state, then it will get crossed back on the other side. Equation (2.7) is the so-called kinematical singularity axiom and is the crossed version of the fact that the form factor has a singularity once an outgoing and an incoming particle have the same rapidity.

These axioms are the form factor counterpart of the S-matrix bootstrap and similarly allow for an exact explicit solution. Indeed, the form factor axioms have been solved exactly for numerous relativistic integrable quantum field theories, [45, 46] including ones with nondiagonal scattering [30, 31].

If we again would be content with neglecting wrapping corrections, the finite volume form factors can be expressed in a very simple way through the infinite volume ones [47]

$$
\left\langle 0|\mathcal{O}(0)| \theta_{1}, \ldots, \theta_{n}\right\rangle_{L}=\frac{1}{\sqrt{\rho_{n} \cdot \prod_{i<j} S\left(\theta_{i}, \theta_{j}\right)}} \cdot F_{n}\left(\theta_{1}, \ldots, \theta_{n}\right) .
$$

Here the finite volume rapidities $\theta_{1}, \ldots, \theta_{n}$ are constrained to obey the Bethe Ansatz quantization condition and $\rho_{n}$ is the Gaudin norm

$$
\rho_{n}=\operatorname{det}\left|\frac{\partial \Phi_{k}}{\partial p_{j}}\right|
$$

which accounts to the difference between the natural finite volume normalization and the continuum normalization in infinite volume. Finally, the square root of the product of Smatrices, which is just a phase, ensures that the finite-volume form factor is a completely symmetric function of the rapidities in contrast to the infinite volume one which obeys (2.5).

Thus we see a similar pattern as for the spectral problem - functional equations in infinite volume and a simple passage to finite volume up to wrapping corrections. 
Form factors seem to be a promising framework for OPE coefficients in the special case of so-called HHL (Heavy-Heavy-Light) diagonal 3-point functions, where two operators correspond to a specific multiparticle state (with large anomalous dimension at strong coupling) while the light operator does not carry any conserved R-charges. In this case the strong coupling classical formula ([48, 49] modified in [44]) denoted schematically by

$$
C_{H H L} \sim \int_{\text {Moduli }} \int d^{2} \sigma V_{L}\left[X^{I}\left(\sigma^{a}\right)\right]
$$

coincides exactly with a classical computation of a 'diagonal' form factor (here we integrate over the moduli space of the classical 2-point correlation function solution of the Heavy operator). This has a distinctive pattern of finite volume dependence (a bit more complicated than (2.8) due to diagonality and disconnected terms. See [44, 50] for details). However this hypothesis has been so far tested only at strong coupling.

The form factor formulation in the context of OPE coefficients has both significant advantages as well as disadvantages. On the positive side, through the existence of infinite volume axioms and simple finite volume reduction, they have the potential to work at any coupling up to wrapping corrections. On the negative side, they are potentially applicable only if the initial and final volume remain the same (i.e. the third 'light' operator does not carry any $J$ charge), and probably only if the two 'heavy' operators are conjugate to each other. This is not a generic situation as typically we have $J_{1}+J_{2}=J_{3}$ with all $J_{i}$ 's distinct from zero. The case $J_{i}=0$ is an important albeit very special case. Another difficulty with the form factor formulation is that the three gauge theory operators are treated very asymetrically. Two gauge theory operators are considered as external multiparticle in and out states, while the third operator is represented by a specific 'effective' worldsheet vertex operator which corresponds, in the form factor language, to a particular solution of the form factor axioms. It is for the moment a-priori not clear how to associate the specific solution of form factor axioms to a particular gauge theory operator/massive string state. Of course this is still premature as currently we do not have at our disposal any solution of the form factor axioms in the case of the worldsheet $A d S_{5} \times S^{5}$ string theory, which remains an outstanding open problem.

In this paper we will pursue an alternative formulation which involves the study of the cubic (light-cone) string field theory vertex. An $A d S_{5} \times S^{5}$ string diagram corresponding to a 3-point correlation function has the topology of two strings joining into a third one and certainly involves the cubic vertex as an essential ingredient. In fact this line of approach was widely used in the pp-wave limit with a formula of the kind

$$
C_{123}=f\left(\Delta_{1}, \Delta_{2}, \Delta_{3}\right) \cdot\langle 1|\langle 2|\left\langle 3 \mid V_{3}\right\rangle_{p p-\text { wave }}
$$

where $\langle i|$ represent the appropriate BMN operators described using pp-wave string excitations and $\left|V_{3}\right\rangle_{p p-w a v e}$ is the pp-wave cubic string vertex constructed in [14-16, 18]. Various concrete formulas were put forward $[9,18,19]$ but we do not have currently a clear generalization of this formula to the full $A d S_{5} \times S^{5}$ context. $^{2}$

\footnotetext{
${ }^{2}$ Some problems were recently encountered in [51].
} 
Leaving this issue aside, in this paper we will concentrate on proposing an integrable approach for computing the cubic vertex

$$
\langle 1|\langle 2|\left\langle 3 \mid V_{3}\right\rangle_{X}
$$

which would be applicable in principle for a curved background $X$ such that the worldsheet string QFT is integrable. Our formulation is a-priori restricted up to wrapping corrections relative to the sizes of the three closed strings. ${ }^{3}$ We will provide functional equations for the above quantities (2.12) in a certain decompactification limit (to be defined later in the paper) and provide a recipe for obtaining the physical finite volume version of (2.12) along the lines of the relation (2.8).

Since we will not control the overall normalization of the vertex in this paper, the function $f\left(\Delta_{1}, \Delta_{2}, \Delta_{3}\right)$ may be incorporated into the vertex so the functional equations may be potentially interpreted as functional equations directly for the OPE coefficients (although this interpretation should be treated with care as the relation between the $A d S_{5} \times$ $S^{5}$ vertex

$$
\langle 1|\langle 2|\left\langle 3 \mid V_{3}\right\rangle_{A d S_{5} \times S^{5}}
$$

and the OPE coefficient $C_{123}$ may will be of a more general form than (2.11)).

The chief obstacle in defining the string vertex for an interacting worldsheet theory is that the hitherto applied constructions of the string field theory vertex used in an essential way mode expansions of the worldsheet fields and operator continuity conditions [14, 21]. In the interacting context we do not have such tools at our disposal ${ }^{4}$ so we apply a form of an integrable bootstrap approach by isolating a decompactification limit allowing to define functional equations incorporating crossing and a subsequent finite volume reduction which should be straightforward as long as we are neglecting wrapping corrections. We thus adopt the same philosophy which was so successful both in the case of the spectral problem and for form factors in relativistic theories.

In the remaining part of the paper we will first recall some information about the pp-wave string field theory and its exact solution, then define the decompactified vertex and propose the string vertex functional equations. Then we will analyze these equations in the case of the massive free boson and compare with the pp-wave results in order to get insight into the required analyticity structure of the solution. In particular we will show how the very nontrivial special functions appearing in the exact pp-wave solution of [52] can be obtained easily from our functional equations. Then we will proceed to define the program for the finite volume reduction and give the string vertex axioms in the general nondiagonal case. Finally, we will also show that some of the general properties of our axioms can be observed in direct weak coupling computations of OPE coefficients in the $s u(2)$ and $s u(1 \mid 1)$ sectors. We relegate various technical details to the appendices.

\footnotetext{
${ }^{3}$ Although wrapping corrections for a single string may be incorporated in this approach.

${ }^{4}$ Although expansions into Zamolodchikov-Faddeev operators may in principle exist, they seem to be impossible to control even in the simplest interacting contexts.
} 

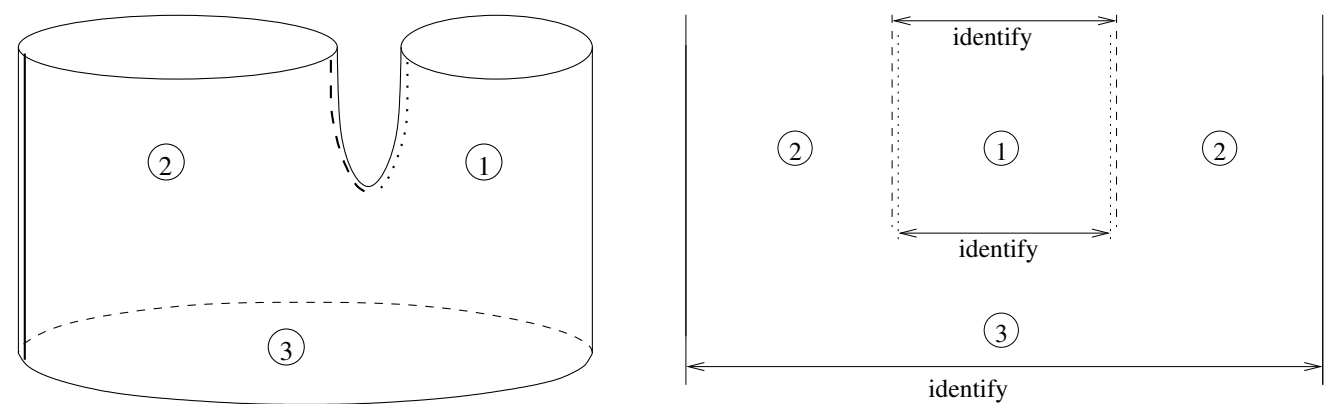

Figure 2. The geometry of the worldsheet for the cubic light cone string field theory vertex in two different representations. The embedded left figure is flattened on the right by cutting along the various lines, which are identified on the right picture.

\section{The pp-wave light cone string field theory vertex}

A unique feature of the pp-wave limit of $A d S_{5} \times S^{5}$ is that when the Green-Schwarz superstring action is considered in an appropriate light-cone gauge, the worldsheet theory reduces to a set of noninteracting massive boson and fermion fields. Hence in this geometry the superstring can be easily quantized exactly [20]. Similarly, the light cone string field theory vertex can also be formulated in a direct generalization of the well known flat space case [21] (although there are several significant subtleties in implementing target space supersymmetry $[15,17,21]$ in order to determine the so-called 'prefactor' part of the SFT vertex).

The light cone string field theory cubic vertex describes the splitting (or joining) of an incoming string into two outgoing strings (see figure 2 (left)). The sizes of the strings, which are proportional to conserved charges add up, hence we have

$$
L_{1}+L_{2}=L_{3}
$$

(or $J_{1}+J_{2}=J_{3}$ - we will often identify the $J$ charge with the size of the cylinder and use one or the other notation depending on whether we want to be closer to the $A d S_{5} \times S^{5}$ string context or whether we want to emphasize a generic integrable QFT point of view).

The pp-wave vertex consists of two distinct parts. One is a universal exponential part which follows from putting the worldsheet QFT onto the geometry shown in the right of figure 2, while the second part is the so-called 'prefactor' which is an operator inserted at the splitting point, and takes the form of a quadratic polynomial in creation and annihilation operators. The latter part is required by target-space supersymmetry algebra, while the former part basically implements just the continuity equations for the worldsheet QFT at the string splitting. Here we will concentrate the discussion on this universal part, although our methods should be applicable also to the full vertex.

Technically, the (bosonic) universal exponential part of the vertex is obtained in the following way. The free massive boson is expanded into cosine and sine modes in the three regions corresponding to strings $\# 1$, \#2 and \#3, with coefficients being the appropriate creation and annihilation operators of the modes. Then one requires the continuity of $\phi$ 
and $\Pi \equiv \partial_{\tau} \phi$ at the string splitting time to obtain linear relations between the relevant creation and annihilation operators:

$$
\sum_{r=1}^{3} \frac{X_{n m}^{r}}{\sqrt{\omega_{m}^{r}}}\left(a_{m}^{+(r)}-a_{m}^{(r)}\right)=0 ; \quad \sum_{r=1}^{3} \operatorname{sgn}_{r} X_{n m}^{r} \sqrt{\omega_{m}^{r}}\left(a_{m}^{+(r)}+a_{m}^{(r)}\right)=0 .
$$

In the above formula $a_{m}^{+(r)}$ is the creation operator for string $r$ with mode number $m, \omega_{m}^{r}$ is proportional to the energy of that mode (see below), while $X_{n m}^{r}$ is a purely geometric overlap between mode $m$ on string $r$ and modes defined on the whole interval (and thus coinciding with modes of string \#3). $\operatorname{sgn}_{r}$ is a sign which is opposite for ingoing and outgoing strings.

The above equations are implemented as operator equations acting on a state $|V\rangle \in$ $\mathcal{H}_{1} \otimes \mathcal{H}_{2} \otimes \mathcal{H}_{3}$ which represents the SFT vertex. The simplest solution of these equations is an exponential of a quadratic form in the creation operators:

$$
|V\rangle=\exp \left\{\frac{1}{2} \sum_{r, s=1}^{3} \sum_{n, m} \bar{N}_{n m}^{r s} a_{n}^{+(r)} a_{m}^{+(s)}\right\}|0\rangle .
$$

The coefficients $\bar{N}_{n m}^{r s}$ are the famous Neumann coefficients ${ }^{5}$ and the problem of finding their explicit form is surprisingly intricate. This comes from the fact that they involve finding the inverse of an infinite dimensional matrix defined through (3.2). In the case of the pp-wave, the solution has been found in two steps. Firstly, the Neumann coefficients where shown to obey a factorization property:

$$
\bar{N}_{n m}^{r s}=-\frac{m n \alpha}{1-4 \mu \alpha K} \frac{\bar{N}_{m}^{r} \bar{N}_{n}^{s}}{\alpha_{s} \omega_{n}^{r}+\alpha_{r} \omega_{m}^{s}}
$$

where

$$
\alpha_{1}=\frac{J_{1}}{J_{3}} ; \quad \alpha_{2}=\frac{J_{2}}{J_{3}} ; \quad \alpha_{3}=-1 ; \quad \alpha=\alpha_{1} \alpha_{2} \alpha_{3}
$$

and $\mu$ is a parameter of the pp-wave background while $K$ and the Neumann vector $\bar{N}_{m}^{r}$ are the nontrivial quantities. Then the Neumann vectors and $K$ have been ultimately determined in the impressive works [53] and [52]. The latter paper (to which we will often refer by the shorthand LSNS) provides a very explicit form for the exact answer which we will discuss at length in section 5 .

Before we finish this section with some comments, let us emphasize that the standard approach to the string field theory vertex outlined above is almost impossible to generalize to the case of an interacting worldsheet QFT (as would be the case for $A d S_{5} \times S^{5}$ ). In the interacting case, we do not have a workable analog of mode expansions hence it is extremely difficult to imagine how to implement continuity relations. Moreover, the above formulation using integer mode numbers seems to be intrinsically tied to a finite volume

\footnotetext{
${ }^{5}$ The bar in $\bar{N}_{n m}^{r s}$ comes form the fact that we are dealing here with cosine and sine modes. When we pass to modes with definite worldsheet momentum, which will be the case relevant for this paper, we will use unbarred notation.
} 


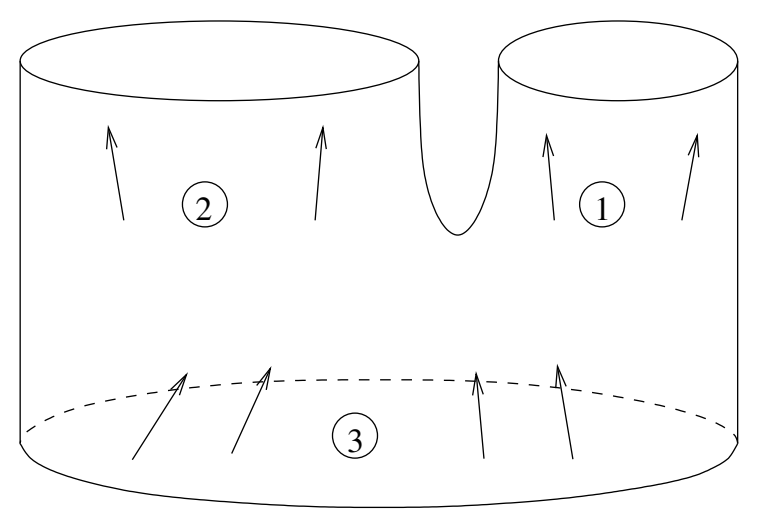

Figure 3. The string field theory vertex with some incoming and outgoing particles in each of the three strings.

setup which makes matters even more complicated. The goal of this paper is to find an alternative approach which bypasses these problems.

Let us now comment on various properties and features of the pp-wave SFT vertex which will be important for our subsequent considerations.

The form of the exponential vertex (3.3) provides for us a clear physical interpretation of the Neumann coefficients. $\bar{N}_{n m}^{r s}$ is just the amplitude of a free massive scalar theory on the pants diagram (figure 2 (right)) with just two particles/modes — one on string $r$ with mode number $n$, the other on string $s$ with mode number $m$, and vacuum on the remaining string(s). The exponential form of the vertex (3.3) means essentially that all amplitudes with a higher number of particles distributed on the pants diagram, see figure 3 , are expressible in a simple way in terms of the 2-particle ones (i.e. in terms of the Neumann coefficients). We expect that in the interacting case the relation between the amplitudes with higher number of particles and lower ones will be less trivial so the goal of formulating the vertex corresponds to finding (equations for) amplitudes with all possible distributions of particles among the three strings and not just generalizing Neumann coefficients to the interacting case.

Let us now introduce some general notation for a generic SFT amplitude with particles with rapidities ${ }^{6} \theta_{1}, \ldots, \theta_{n}$ on string $\# 3, \theta_{1}^{\prime}, \ldots, \theta_{m}^{\prime}$ on string $\# 2$ and $\theta_{1}^{\prime \prime}, \ldots, \theta_{l}^{\prime \prime}$ on string \#1. We also explicitly mark the sizes of the respective strings:

$$
\mathbf{N}_{L_{3} \mid L_{2} ; L_{1}}^{3 \mid 2 ; 1}\left(\theta_{1}, \ldots, \theta_{n} \mid \theta_{1}^{\prime}, \ldots, \theta_{m}^{\prime} ; \theta_{1}^{\prime \prime}, \ldots, \theta_{l}^{\prime \prime}\right)
$$

For the case of the pp-wave SFT vertex, these quantities can be directly expressible in terms of the Neumann coefficients. Assuming for the moment the absence of the prefactor, we would have

$$
\mathbf{N}_{L_{3} \mid L_{2} ; L_{1}}^{3 \mid 2 ; 1}\left(\theta_{1}, \theta_{2} \mid \varnothing ; \varnothing\right) \equiv N_{n_{1} n_{2}}^{33} \quad \mathbf{N}_{L_{3} \mid L_{2} ; L_{1}}^{3 \mid 2 ; 1}\left(\theta_{1} \mid \theta_{2} ; \varnothing\right) \equiv N_{n_{1} n_{2}}^{32}
$$

\footnotetext{
${ }^{6}$ For the sake of clear notation here we parametrize the particles by relativistic rapidities, but the definitions will go over verbatim either to a parametrization in terms of momenta or in terms of complex AdS rapidities.
} 
where $n_{i}$ are mode numbers corresponding to particular momenta/rapidities and the empty set $\varnothing$ just denotes the vacuum. A more complicated example is

$$
\mathbf{N}_{L_{3} \mid L_{2} ; L_{1}}^{3 \mid 2 ; 1}\left(\theta_{1}, \theta_{2} \mid \theta_{3} ; \theta_{4}\right) \equiv N_{n_{1} n_{2}}^{33} N_{n_{3} n_{4}}^{12}+N_{n_{1} n_{3}}^{32} N_{n_{2} n_{4}}^{31}+N_{n_{2} n_{3}}^{32} N_{n_{1} n_{4}}^{31}
$$

When we give formulas for the pp-wave case, we will alternatively use the conventional notation of Neumann coefficients, but always recall (3.7).

Another interesting observation comes from analyzing some important parameter regimes appearing in the pp-wave case. $\mu$ is a parameter which is essentially the inverse of the ' $t$ Hooft coupling. It appears in the frequency of the appropriate mode as

$$
\omega_{m}^{r}=\sqrt{m^{2}+\mu^{2} \alpha_{r}^{2}} .
$$

For our purposes it is convenient to reformulate all formulas by trading the integer mode numbers for physical worldsheet momenta. The momenta are given by $p= \pm 2 \pi m / J_{r}$, and thus the frequency becomes

$$
\omega_{m}^{r}=\frac{\left|\alpha_{r}\right| J}{2 \pi} \sqrt{p^{2}+M^{2}}
$$

where $J \equiv J_{3}$ and the mass of the scalar field is related to $\mu$ through

$$
M=\frac{2 \pi}{J} \mu .
$$

In the pp-wave times, people were mostly interested in comparison with gauge theory perturbative computations and thus concentrating on an expansion around $\mu=\infty$ in inverse powers of $\mu$. In particular they employed simpler asymptotic versions of the Neumann coefficients which neglected terms of the type

$$
e^{-2 \pi \mu\left|\alpha_{r}\right|}
$$

It is interesting to realize that this term, when expressed in terms of the physical mass of the free boson (3.11), becomes

$$
e^{-M J_{r}}
$$

which is exactly the scale of wrapping corrections associated to string $r$. In fact this nicely explains the observation made in [52] about the similarity of the formulas of the leading exponential corrections to the Neumann coefficients with Casimir energy of the free massive boson.

In the following, we will also need expressions corresponding to modes with definite worldsheet momentum - thus the so-called BMN modes instead of the cosine and sine modes used in the derivation of the Neumann coefficients. The explicit relations are given e.g. in [16], in particular we have

$$
N_{m n}^{r s}=\frac{1}{2}\left(\bar{N}_{m n}^{r s}-\bar{N}_{-m-n}^{r s}\right)
$$

for positive mode numbers. 
We will be mostly, but as it will turn out not exclusively, concentrated on the string vertex when neglecting wrapping corrections. In this limit, the expression for $N_{m n}^{r s}$ no longer involves special functions but is still apparently quite cumbersome [53]:

$$
N_{m n}^{r s} \propto\left[\frac{\sqrt{\left(\omega_{m}^{r}+\mu \alpha_{r}\right)\left(\omega_{n}^{s}+\mu \alpha_{s}\right)}}{\omega_{m}^{r}+\omega_{n}^{s}}-\frac{\sqrt{\left(\omega_{m}^{r}-\mu \alpha_{r}\right)\left(\omega_{n}^{s}-\mu \alpha_{s}\right)}}{\omega_{m}^{r}+\omega_{n}^{s}}\right] s_{r m} s_{s n}
$$

with

$$
s_{1 m}=s_{2 m}=1 \quad s_{3 m}=-2 \sin \left(\pi m \alpha_{1}\right) .
$$

Surprisingly enough, once we parametrize the modes by rapidities $p=M \sinh \theta$, the above expression simplifies drastically: ${ }^{7}$

$$
N^{33}\left(\theta, \theta^{\prime}\right)_{\text {asympt }} \propto-2 \frac{\sin \frac{p L_{1}}{2} \sin \frac{p^{\prime} L_{1}}{2}}{\cosh \frac{\theta-\theta^{\prime}}{2}}
$$

where we extracted simple factors related to the normalization condition for the modes and some overall constant factor. The subscript asympt denotes the fact that we neglected all exponential wrapping corrections in $e^{-M L_{1}}$.

Let us make some comments on the above expression (3.17). Firstly, we see that the discrete nature of the finite volume integer modes does not play here any important role. In fact the above expression is extremely simple when expressed in terms of infinite volume rapidities. The passage to finite volume amounts here ${ }^{8}$ just to evaluating the above expression (3.17) for rapidities corresponding to quantized momenta i.e. $p=M \sinh (\theta)=2 \pi n / L$. This is in direct correspondence with the finite volume evaluation of form factors (2.8).

Secondly, the analytic structure of this function is also quite appealing as there is a pole at $\theta=\theta^{\prime}+i \pi$, which is exactly the characteristic position of the so-called kinematical singularity for form factors, with the $i \pi$ intimately related to crossing properties.

Thirdly, there are nevertheless still some surprising features of the expression (3.17). Two-particle form factors typically have vanishing residue at the kinematical pole (see $(2.7)$ ), while here ${ }^{9}$ the residue is nonzero and is in fact quite bizarre. The $\sin \frac{p L}{2}$ factors are also quite surprising by themselves. They are almost of the 'wrapping' type, however instead of being exponentially suppressed, they are oscillatory.

Moreover, if one would consider the asymptotic form of $N^{32}$

$$
N^{32}\left(\theta, \theta^{\prime}\right)_{\text {asympt }} \propto \frac{\sin \frac{p L_{1}}{2}}{\sinh \frac{\theta-\theta^{\prime}}{2}}
$$

one would see that (3.17) and (3.18) are related by a surprisingly modified form of crossing relation

$$
N^{33}\left(\theta, \theta^{\prime}-i \pi\right)_{\text {asympt }}=-2 i \sin \frac{p^{\prime} L_{1}}{2} N^{32}\left(\theta, \theta^{\prime}\right) \text { asympt }
$$

\footnotetext{
${ }^{7}$ We provide more formulas and discuss various intriguing features of this limit in section 5.3.

${ }^{8}$ Recall that we are always neglecting exponential wrapping corrections.

${ }^{9}$ Recall from the discussion above that Neumann coefficients can be interpreted as two particle amplitudes.
} 

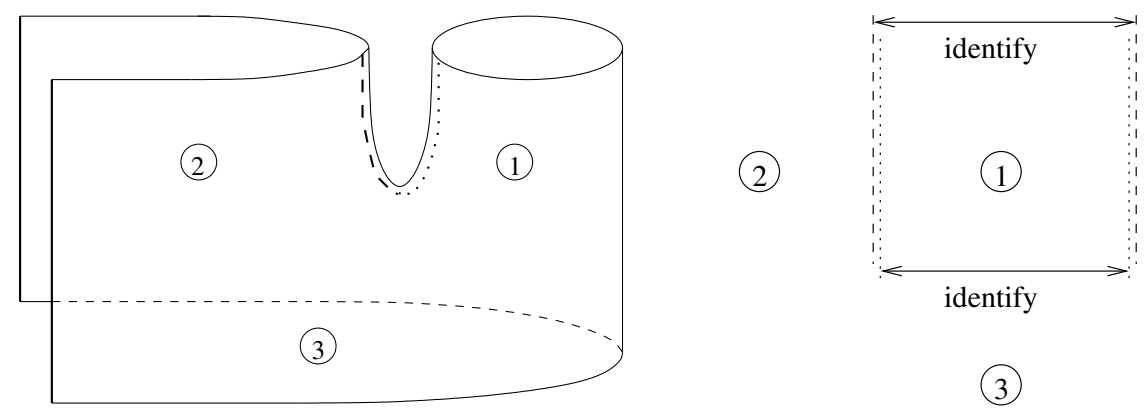

Figure 4. The decompactified string field theory vertex in two different geometrical representations.

which, incidentally bears a striking resemblance to the modified crossing observed in $[54,55]$.

So to conclude this section, we see that the asymptotic form of the pp-wave Neumann coefficients very strongly suggests the existence of an infinite volume formulation based on analyticity properties such as crossing, kinematical singularity etc. In the remaining part of the paper we will indeed provide such a formulation and also show that the apparent modification of crossing in (3.19) is in fact an artefact of the large volume limit and the true crossing property should be different.

\section{The decompactified string vertex and the SFT axioms}

As emphasized before, in order to be able to formulate functional equations incorporating crossing property it is crucial to define a decompactified version of the SFT vertex. We show such a construction in figure 4 , where we cut strings \#2 and \#3 and extended their boundaries to infinity. The right hand side of this figure shows the resulting pattern of identifications in the two-dimensional plane. Here string \#1 remains of finite size $L \equiv L_{1}$ and there will be a nontrivial dependence on the dimensionless product $m L$. In particular, we expect that the decompactified vertex amplitudes will incorporate all wrapping corrections associated with string \#1.

Of course, we could have just as well made the cut along string \#1 and \#3, leaving string \#2 at finite size. In fact in order to find the physical finite volume SFT vertex from integrability we will advocate considering simultaneously both possibilities, solving the associated two sets of functional equations and then requiring that the finite volume reductions of both solutions will coincide. We will describe this in more detail in section 7 .

Finally, we note that unfortunately we cannot decompactify both outgoing strings, because then the ingoing string \#3 would split into two disconnected pieces. In fact we will find that the size of the leftover finite size string $L$ will play a crucial role in formulating the SFT axioms.

\subsection{The decompactified SFT axioms}

In the following we cut string \#2 and string \#3 and extend their boundaries to infinity as shown on the left of figure 2. On the right, one can see the full infinite spacetime domain 
of string \#3 on the lower part, while the infinite spacetime domain of string \#2 with a missing strip of size $L$ on the upper part of the figure. The two sides of the strip are identified to form the space-time cylinder of string \#1. The other identification on the figure makes the space-time for string \#2 continous, i.e. leaving from left to right on the left of the strip we appear immediately on the right of the strip.

The aim of this section is to propose functional equations for the amplitude with prescribed number and momenta of particles on the decompactified strings \#2 and \#3, while the particle content in the compact string \#1 may be arbitrary. Since these string \#1 excitations will not enter the equations at all, we will denote them by $\bullet$ below.

As the decompactified SFT vertex amplitude has slightly different properties ${ }^{10}$ from the finite volume one discussed in section 3, we introduce some specific notation in this case. We thus denote the decompactified SFT vertex amplitude by

$$
\mathbf{N}_{\bullet, L}^{3 \mid 2}\left(\theta_{1}, \ldots, \theta_{n} \mid \theta_{1}^{\prime}, \ldots, \theta_{m}^{\prime}\right)
$$

which contains particles with rapidities $\left\{\theta_{i}\right\}$ in domain \#3 and with rapidities $\left\{\theta_{j}^{\prime}\right\}$ in domain \#2.

The superscripts denote the noncompact ingoing and outgoing strings, $L$ is the size of the remaining closed string \#1 and $\bullet$ denotes its specific state as well as any local operator inserted at the string splitting point (like the prefactor in the pp-wave SFT vertex), see figure 5 for a graphical notation.

In the following, we will sometimes suppress the lower subscript $\bullet, L$ as long as it remains unchanged. We assume that the particles scatter on each other diagonally with the scattering matrix $S\left(\theta_{i}, \theta_{j}\right)$. We will cover the general non-diagonal case in section 6 . This S-matrix does not necessarily depend on the differences of the rapidities but satisfies unitarity

$$
S\left(\theta_{i}, \theta_{j}\right)=S\left(\theta_{j}, \theta_{i}\right)^{-1} .
$$

When particles pass through each other they scatter with the S-matrix, thus their ordering is essential. States in domain $\# 3$ are preparated at $t=-\infty$ and contain particles with ordered rapidities $\theta_{i}>\theta_{i+1}$, i.e. the fastest is on the leftmost. We call these states initial states. States in domain \#2 contain ordered particles with rapidities $\theta_{i+1}^{\prime}<\theta_{i}^{\prime}$, in which the fastest is the rightmost. These states are called final states. The coefficient $\mathbf{N}_{\bullet}^{3 \mid 2}$ above describes the transition amplitude from an initial to a final state. Clearly if there were no space deficiency, $L=0$, (and trivial operator insertion), $\mathbf{N}_{\bullet}^{3 \mid 2}$ would be nothing but the scattering matrix element, nonvanishing only for coinciding sets of rapidities. If, however, $L \neq 0$, or there is an operator insertion the corresponding $\mathbf{N}_{\bullet}^{3 \mid 2}$ is similar to a form factor: that is to a matrix element of an operator. This operator is local for $L=0$ but is non-local for $L \neq 0$. In the following we focus on the $L \neq 0$ case. This is similar to the situation, when we analyze the form factors of an operator, which is nonlocal with respect to the particles. Moving a particle around the space deficiency would pick up a phase factor

\footnotetext{
${ }^{10}$ This is exactly as for the relation between finite volume and infinite volume form factors which differ by a Jacobian factor and a product of S-matrices neccessary to ensure symmetry of the finite volume one cf. (2.8).
} 


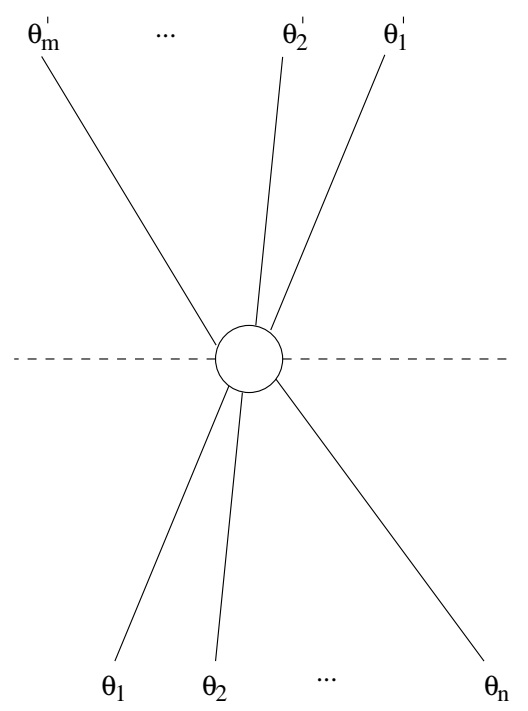

Figure 5. Graphical notation for the SFT vertex. Domain \#3 is below the dashed line and contains incoming particles $\left\{\theta_{i}\right\}$, while domain \#2 is above the line and contains outgoing particles $\left\{\theta_{i}\right\}$. The emission of string \#1 is represented by the circle, which can be understood as a partly nonlocal operator insertion in the form factor language. It introduces a discontinuity which is distributed symmetrically on the border of the two domains indicated by dashed lines.

proportional both to $L$ and to its momenta: $e^{i p L}$. This bears some similarity with the form factor axioms with nonzero index of mutual locality [56]. Note, however, that here the analogous index is momentum dependent which is a completely novel and unique feature of the string vertex. By the choice of the bases in domains \#3 and \#2 we can freely place this nonlocality wherever we want. To be in accordance with the pp-wave conventions we distribute the nonlocality in an equal way on the border of domains \#3 and \#2, which we indicate by dashed lines on the figures.

This means we define the crossing equations as

$$
\begin{aligned}
& \mathbf{N}_{\bullet, L}^{3 \mid 2}\left(\theta_{1}, \ldots, \theta_{n} \mid \theta_{1}^{\prime}, \ldots, \theta_{m}^{\prime}\right)=e^{i p\left(\theta_{1}^{\prime}\right) \frac{L}{2}} \mathbf{N}_{\bullet, L}^{3 \mid 2}\left(\theta_{1}, \ldots, \theta_{n}, \theta_{1}^{\prime}-i \pi \mid \theta_{2}^{\prime}, \ldots, \theta_{m}^{\prime}\right) \\
& \mathbf{N}_{\bullet, L}^{3 \mid 2}\left(\theta_{1}, \ldots, \theta_{n} \mid \theta_{1}^{\prime}, \ldots, \theta_{m}^{\prime}\right)=e^{-i p\left(\theta_{m}^{\prime}\right) \frac{L}{2}} \mathbf{N}_{\bullet, L}^{3 \mid 2}\left(\theta_{m}^{\prime}+i \pi, \theta_{1}, \ldots, \theta_{n} \mid \theta_{1}^{\prime}, \ldots, \theta_{m-1}^{\prime}\right) .
\end{aligned}
$$

They are represented graphically on figure 6 .

In these equations it is understood that no rapidites in the initial and final states coincide $\theta_{i} \neq \theta_{j}^{\prime}$ as otherwise disconnected terms can arise. ${ }^{11}$ Crossing all particles to domain \#3 we can define the elementary SFT vertex

$$
\mathbf{N}_{\bullet}, L\left(\theta_{1}, \ldots, \theta_{n}\right) \equiv \mathbf{N}_{\bullet, L}^{3 \mid 2}\left(\theta_{1}, \ldots, \theta_{n} \mid \varnothing\right)
$$

which obviously contains all the information. ${ }^{12}$ This vertex is represented graphically on figure 7 .

\footnotetext{
${ }^{11}$ Let us note that it is possible to introduce a sign in both equations (4.3) and (4.4) to accommodate different normalization conventions. This does not change, of course, any physical content.

${ }^{12}$ Recall that the empty set $\varnothing$ denotes the vacuum (no particles) while $\bullet$ stands for any particle content.
} 

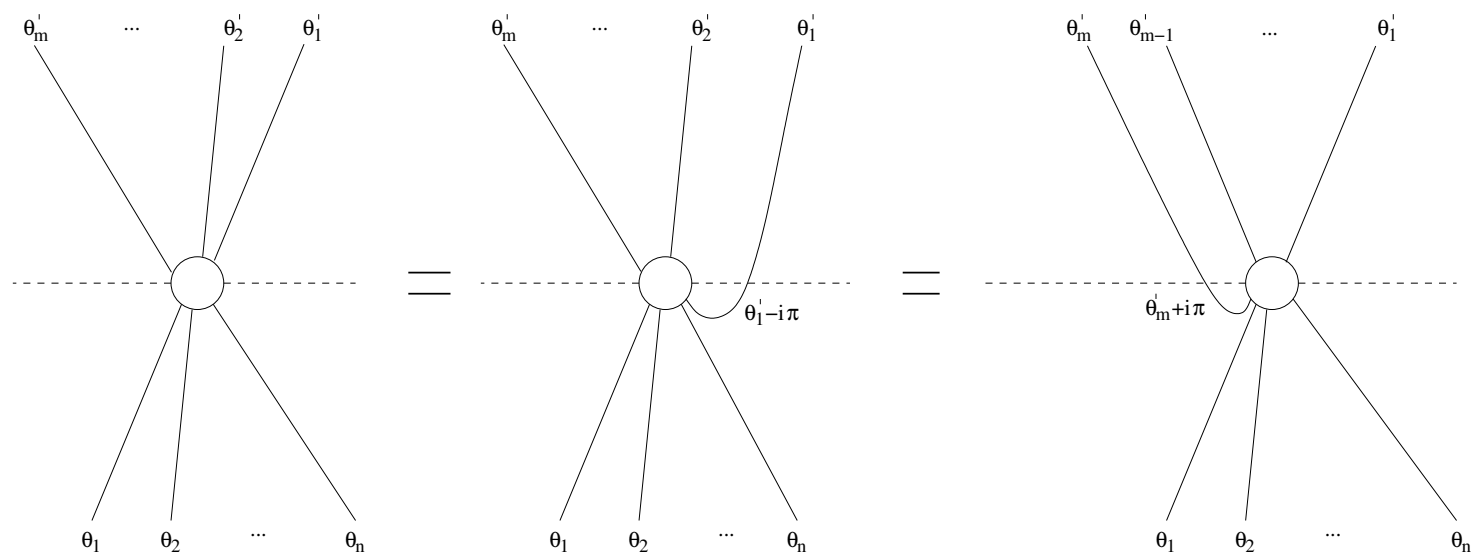

Figure 6. Crossing transformations for the SFT vertex.

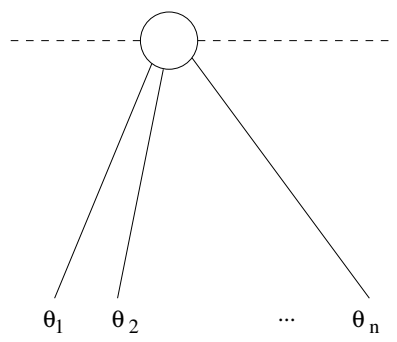

Figure 7. Elementary SFT vertex.
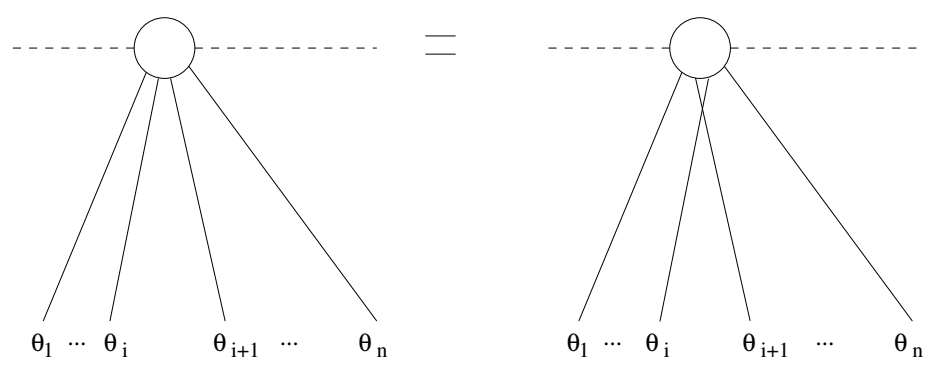

Figure 8. Permutation axiom of the SFT vertex.

Now we formulate the axioms it should satisfy. As the initial state is a scattering state exchanging two neighbouring particles leads to the factor of the scattering matrix, see figure 8:

$$
\mathbf{N}_{\bullet}, L\left(\theta_{1}, \ldots, \theta_{i}, \theta_{i+1}, \ldots, \theta_{n}\right)=S\left(\theta_{i}, \theta_{i+1}\right) \mathbf{N}_{\bullet}, L\left(\theta_{1}, \ldots, \theta_{i+1}, \theta_{i}, \ldots, \theta_{n}\right) .
$$

Crossing the first particle to domain \#2 and crossing back to the last position we obtain the monodromy relation

$$
\mathbf{N}_{\bullet}, L\left(\theta_{1}, \theta_{2}, \ldots, \theta_{n}\right)=e^{-i p\left(\theta_{1}\right) L} \mathbf{N}_{\bullet}, L\left(\theta_{2}, \ldots, \theta_{n}, \theta_{1}-2 i \pi\right)
$$

which expresses the nonlocality of the "operator insertion", see figure 9. Here we used that $p\left(\theta_{1}+i \pi\right)=-p\left(\theta_{1}\right)$. 

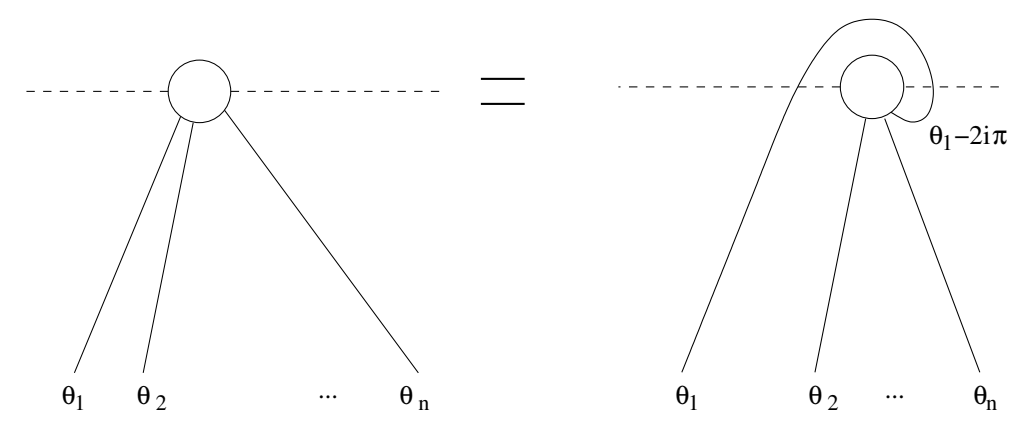

Figure 9. Monodromy axiom of the SFT vertex.

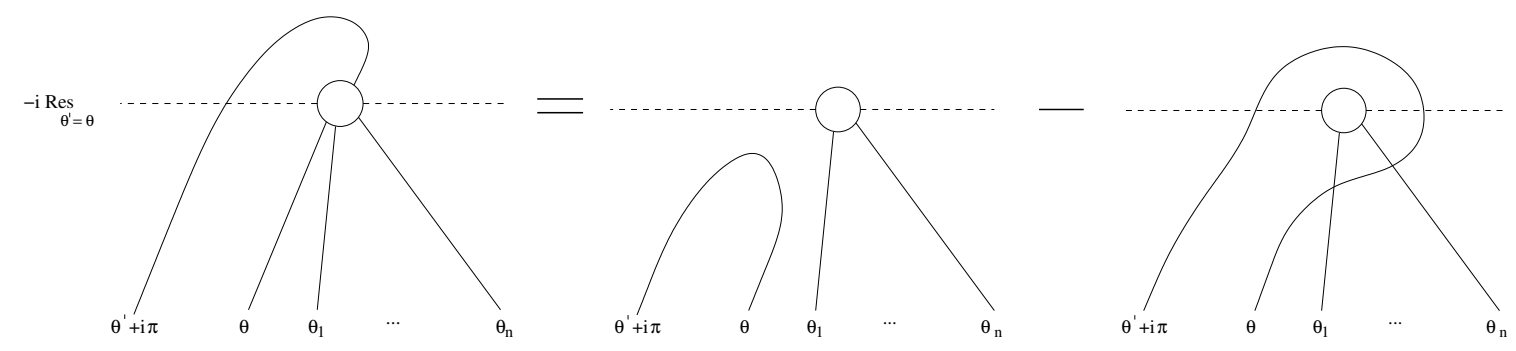

Figure 10. Kinematical singularity axiom of the SFT vertex.

The above two relations provide functional equations which enable to determine the coefficient $\mathbf{N}_{\bullet}, L$ once its analytical structure is known. $\mathbf{N}_{\bullet}, L$ must be a meromorphic function of the rapidites, whose poles have physical origins. There are poles which have kinematical and others which have dynamical origins. A kinematical singularity can appear whenever, after crossing, an initial particles' rapidity coincides with a final one. The residue of the pole is proportional to the amplitude where the two particles are missing as:

$$
-i \operatorname{Res}_{\theta^{\prime}=\theta} \mathbf{N}_{\bullet}, L\left(\theta^{\prime}+i \pi, \theta, \theta_{1}, \ldots, \theta_{n}\right)=\left(1-e^{i p(\theta) L} \prod_{i=1}^{n} S\left(\theta, \theta_{i}\right)\right) \mathbf{N}_{\bullet}, L\left(\theta_{1}, \ldots, \theta_{n}\right) .
$$

The proportionality factor expresses the fact that the on-shell particle can pass the other particles and the defect on both sides as shown on figure 10 .

The dynamical singularity axiom is related to the existence of boundstates and expresses the SFT vertex of the boundstate in terms of that of the fundamental particles. As this axiom does not provide any restriction on the elementary SFT vertex we do not write out explicitly here, but spell out the details in the nondiagonal case in section 6 below.

Let us finish this part by specifying the kinematical singularity axiom for the case when there are particles both in the initial and final states, as this equation will show up in the weak coupling limit of the OPE coefficients:

$$
\begin{gathered}
-i \operatorname{Res}_{\theta^{\prime}=\theta} \mathbf{N}_{\bullet, L}^{3 \mid 2}\left(\theta, \theta_{1}, \ldots, \theta_{n} \mid \theta_{1}^{\prime}, \ldots, \theta_{m}^{\prime}, \theta^{\prime}\right)=e^{-i p(\theta) L / 2}\left(1-e^{i p(\theta) L} \prod_{i=1}^{n} S\left(\theta, \theta_{i}\right) \prod_{j=1}^{n} S\left(\theta_{j}^{\prime}, \theta\right)\right) \times \\
\mathbf{N}_{\bullet}^{3 \mid 2}\left(\theta_{1}, \ldots, \theta_{n} \mid \theta_{1}^{\prime}, \ldots, \theta_{m}^{\prime}\right)
\end{gathered}
$$


Finally, note that the above equations do not depend in any way on the state of the compact string (string \#1 here). This is in fact very natural and is analogous to the well known form factor axioms which have exactly the same form for any local operator. The form factor axioms do not have a unique solution, however, and various solutions correspond to form factors of various local operators. We expect the situation to be similar here - the axioms for $\mathbf{N}_{\bullet}, L\left(\theta_{1}, \ldots, \theta_{n}\right)$ will have many solutions depending on the particle content of string \#1 and on the choice of prefactor operator in the SFT vertex inserted at the splitting point.

However, this time in contrast to the ordinary form factor case, we will be able to provide additional information which will severely restrict the dependence on the string \#1 state. This will be discussed in detail in section 7 , where we complete the formulation of our program for the SFT vertex.

\section{The free massive boson example (or the pp-wave SFT vertex)}

In this section we will discuss the simplest case for which our integrable approach should work i.e. a free massive boson. This is precisely the case of the pp-wave SFT vertex, ${ }^{13}$ the Neumann coefficients of which are known exactly.

The consideration of the pp-wave vertex is interesting for a variety of reasons. Firstly, we may check that the proposed booststrap axioms are indeed satisfied. Secondly, we may analyze the analytical structure of the pp-wave Neumann coefficients to put forward some 'empirical' analyticity requirements for the solutions of the bootstrap SFT vertex axioms in the general interacting case. Thirdly, we may investigate directly their asymptotic limit neglecting wrapping corrections, which limit turns out to have surprisingly subtle properties. Finally, we may see how to reconstruct the exact (decompactified) pp-wave Neumann coefficients directly from our axioms together with the analyticity assumptions mentioned above.

\subsection{A review of LSNS formulas}

Let us start by reviewing the known exact solution for the pp-wave Neumann coefficients as given by [52]. These formulas involve quite a lot of notation and new special functions $\Gamma_{\mu}(z)$ introduced by LSNS, whose properties and definitions we recall in appendix B. We also pass here to rapidity variables instead of integer mode numbers and introduce some modifications of the special functions - which we denote by $\tilde{\Gamma}_{\mu}(\theta)$ - which are more convenient for our purposes.

Recall from section 3 that the Neumann coefficients in the cosine-basis have the structure

$$
\bar{N}_{m n}^{r s}=\rho \frac{m n}{\alpha_{s} \omega_{m}^{r}+\alpha_{r} \omega_{n}^{s}} \bar{N}_{m}^{r} \bar{N}_{n}^{s}
$$

\footnotetext{
${ }^{13}$ Since the treatment of massless particles in the integrable S-matrix language is in general quite subtle, together with the importance of wrapping, a discussion of the flat space SFT vertex of [21] would require a lot of care.
} 
where we focused on the dependence on the quantization numbers $n, m$ and put the rest into the normalization constant $\rho$. The Neumann vectors $\bar{N}_{m}^{r}$ are defined in terms of the function $f_{m}^{(r)}$ as

$$
\bar{N}_{m}^{r}=\sqrt{\frac{\omega_{m}^{r}}{m}} \frac{\omega_{m}^{r}+\alpha_{r} \mu}{\alpha_{r} m} f_{m}^{(r)} .
$$

The Neumann matrix above is related to the cosine basis. The coefficients for the sine basis can be obtained as

$$
\bar{N}_{-m-n}^{r s}=-\frac{\omega_{m}^{r}-\alpha_{r} \mu}{m} \frac{\omega_{n}^{s}-\alpha_{s} \mu}{n} \bar{N}_{m n}^{r s}
$$

The Neumann coefficients in the exponential basis relevant for our considerations follow through (3.14)

$$
N_{m n}^{r s}=\frac{1}{2}\left(1+\frac{\omega_{m}^{r}-\alpha_{r} \mu}{m} \frac{\omega_{n}^{s}-\alpha_{s} \mu}{n}\right) \bar{N}_{m n}^{r s} .
$$

As we explained earlier, the rapidity parametrization considerably simplifies the formulas. Thus we express $\omega_{m}^{r}$ and the mode number $m$ in terms of the rapidity $\theta_{m}$ as:

$$
\omega_{m}^{r}=\sqrt{m^{2}+\alpha_{r}^{2} \mu^{2}}=\left|\alpha_{r}\right| \mu \cosh \theta_{m} ; \quad m=\left|\alpha_{r}\right| \mu \sinh \theta_{m} .
$$

In the following we introduce formulas, which are valid for any signs of $\alpha_{r}$. However, the expressions will depend on this sign, which we denote by $\operatorname{sgn}_{r}$. Using the formulas above we get

$$
\frac{\omega_{m}^{r}-\alpha_{r} \mu}{m} \frac{\omega_{n}^{s}-\alpha_{s} \mu}{n}=\left(\tanh \frac{\theta_{m}}{2}\right)^{\operatorname{sgn}_{r}}\left(\tanh \frac{\theta_{n}}{2}\right)^{\operatorname{sgn}_{s}}
$$

and the general Neumann matrix can be written as:

$$
\begin{aligned}
N_{m n}^{r s}= & \frac{\rho}{2} \frac{\mu \sinh \theta_{m} \sinh \theta_{n}}{\operatorname{sgn} \cosh _{m}+\operatorname{sgn}_{r} \cosh \theta_{n}} \frac{1}{\alpha_{s} \alpha_{r}}\left[1+\left(\tanh \frac{\theta_{m}}{2}\right)^{-\operatorname{sgn}_{r}}\left(\tanh \frac{\theta_{n}}{2}\right)^{-\operatorname{sgn}_{s}}\right] \\
& \times \sqrt{\frac{\cosh \theta_{m}}{\sinh \theta_{m}}} \sqrt{\frac{\cosh \theta_{n}}{\sinh \theta_{n}}} f_{m}^{(r)} f_{n}^{(s)}
\end{aligned}
$$

Let us spell out the details in the three distinct cases. For $\alpha_{3}=-1$ we have

$$
N_{m n}^{33}=-\rho \frac{d^{(3)}\left(\theta_{m}\right) d^{(3)}\left(\theta_{n}\right)}{\cosh \frac{1}{2}\left(\theta_{m}-\theta_{n}\right)} ; \quad d^{(3)}\left(\theta_{m}\right)=-\sqrt{\mu} \sinh \frac{\theta_{m}}{2} \sqrt{\frac{\cosh \theta_{m}}{\sinh \theta_{m}}} f_{m}^{(3)}
$$

while for the other cases with $i=1,2$

$$
\begin{aligned}
& N_{m n}^{3 i}=N_{n m}^{i 3}=-\rho \frac{d^{(3)}\left(\theta_{m}\right) d^{(i)}\left(\theta_{n}\right)}{\sinh \frac{1}{2}\left(\theta_{m}-\theta_{n}\right)} ; \quad d^{(i)}\left(\theta_{n}\right)=\frac{\sqrt{\mu}}{\alpha_{i}} \cosh \frac{\theta_{n}}{2} \sqrt{\frac{\cosh \theta_{n}}{\sinh \theta_{n}}} f_{n}^{(i)} \\
& N_{m n}^{i j}=\rho \frac{d^{(i)}\left(\theta_{m}\right) d^{(j)}\left(\theta_{n}\right)}{\cosh \frac{1}{2}\left(\theta_{m}-\theta_{n}\right)} ; \quad i, j=1,2 .
\end{aligned}
$$

The $d^{(r)}(\theta)$ are closely related to the Neumann vectors, but are more convenient in the following. We now analyze the expressions $d^{(r)}(\theta)$ one by one by starting with $d^{(3)}(\theta)$. We 
recall from [52] that ${ }^{14}$

$$
f_{m}^{(3)}=\frac{\sqrt{m}}{\pi} \sin \left(m \pi \alpha_{2}\right) \frac{e^{\tau_{0}\left(\mu-\omega_{m}^{3}\right)}}{\omega_{m}^{3}} \frac{\Gamma_{\mu \alpha_{1}}\left(m \alpha_{1}\right) \Gamma_{\mu \alpha_{2}}\left(m \alpha_{2}\right)}{\Gamma_{\mu}(m)} M\left(0^{+}\right)
$$

where

$$
\tau_{0}=\alpha_{1} \log \alpha_{1}+\alpha_{2} \log \alpha_{2}=\alpha_{1} \log \mu \alpha_{1}+\alpha_{2} \log \mu \alpha_{2}-\log \mu
$$

and $\Gamma_{\mu}(z)$ is defined in appendix B. We move $e^{\tau_{0} \mu} M\left(0^{+}\right)$to $\rho$, as it appears in all $f$ coefficients. Using the renormalized deformed $\tilde{\Gamma}_{\mu}$ functions introduced in appendix B, together with the rapidity parametrization, we obtain

$$
d^{(3)}\left(\theta_{m}\right)=-\frac{\sinh \frac{\theta_{m}}{2} \sin \left(\mu \pi \alpha_{2} \sinh \theta_{m}\right)}{\pi \sqrt{\cosh \theta_{m}}} \frac{\tilde{\Gamma}_{\mu \alpha_{1}}\left(\theta_{m}\right) \tilde{\Gamma}_{\mu \alpha_{2}}\left(\theta_{m}\right)}{\tilde{\Gamma}_{\mu}\left(\theta_{m}\right)}
$$

After a similar manipulation on $d^{(i)}(\theta)$ for $i=1,2$ we can turn

$$
f_{n}^{(i)}=\frac{e^{\tau_{0}\left(\mu+\omega \frac{n}{\alpha_{i}}\right)} \alpha_{i}}{\omega_{\frac{n}{\alpha_{i}}} \sqrt{n} \alpha_{1} \alpha_{2}} \frac{\Gamma_{\mu}\left(\frac{n}{\alpha_{i}}\right)}{\Gamma_{\alpha_{2} \mu}\left(\frac{\alpha_{2} n}{\alpha_{i}}\right) \Gamma_{\alpha_{1} \mu}\left(\frac{\alpha_{1} n}{\alpha_{i}}\right)} M\left(0^{+}\right)
$$

with $\omega_{z}=\sqrt{z^{2}+\mu^{2}}$ into the expression

$$
d^{(i)}\left(\theta_{n}\right)=\frac{1}{2 \mu \sqrt{\alpha_{i}} \alpha_{1} \alpha_{2} \sqrt{\cosh \theta_{n}} \sinh \frac{\theta_{n}}{2}} \frac{\tilde{\Gamma}_{\mu}\left(\theta_{n}\right)}{\tilde{\Gamma}_{\alpha_{2} \mu}\left(\theta_{n}\right) \tilde{\Gamma}_{\alpha_{1} \mu}\left(\theta_{n}\right)} .
$$

These expressions together with equations (5.8)-(5.10) provide the exact finite $L_{i}$ expressions for the Neumann coefficients.

\subsection{The decompactification limit of the LSNS formulas and their analyticity properties}

In order to make contact with the SFT vertex axioms introduced in section 4, let us take the same decompactification limit, in which we send $L_{3}, L_{2} \rightarrow \infty$ and keep $L_{1}=L_{3}-L_{2}$ finite. Moreover we will also keep the mass of the scalar field and the particle rapidities fixed. This entails sending also $\mu$ and the integer mode number $m$ to $\infty$, such that

$$
\frac{2 \pi \mu}{L_{3}} \equiv M \quad \frac{2 \pi m_{i}}{\left|\alpha_{i}\right| L_{3}} \equiv M \sinh \theta
$$

are kept fixed. In this limit

$$
\alpha_{1}=\frac{L_{1}}{L_{3}} \rightarrow 0 ; \quad \alpha_{2} \rightarrow 1
$$

while the quantities

$$
\mu \alpha_{1}=\frac{M L_{1}}{2 \pi} ; \quad m_{3} \alpha_{1}=\frac{p L_{1}}{2 \pi}=\frac{M L_{1} \sinh \theta}{2 \pi}=\mu \alpha_{1} \sinh \theta
$$

\footnotetext{
${ }^{14}$ There is also an extra factor $(-1)^{m+1}$ for $d^{(1)}$, which we choose to be 1 to unify the notation for $d^{(1)}$ and $d^{(2)}$.
} 
stay finite. In the following formulas we will drop the subscript in $L_{1}$ and use the notation $L \equiv L_{1}$ as in the SFT vertex axioms of section 4 .

The key quantities appearing in the Neumann coefficient formulas (5.8)-(5.10) now have the following finite decompactified limits: ${ }^{15}$

$$
d^{(3)}(\theta)=-\frac{\sinh \frac{\theta}{2} \sin \frac{p L}{2}}{\pi \sqrt{\cosh \theta}} \cdot \tilde{\Gamma}_{\frac{M L}{2 \pi}}(\theta) \cdot e^{-\frac{\theta}{2 \pi} p L}
$$

and

$$
d^{(2)}(\theta)=\frac{\pi}{M L \sqrt{\cosh \theta} \sinh \frac{\theta}{2}} \cdot \frac{1}{\tilde{\Gamma}_{\frac{M L}{2 \pi}}(\theta)} \cdot e^{\frac{\theta}{2 \pi} p L}
$$

where here and from now on $p=M \sinh \theta$. We must still address, however, one minor detail. In the decompactified case, the external states are conventionally normalized to a Dirac delta function in rapidities, while the finite volume mode states are normalized to Kronecker deltas in mode numbers. So we have to factor out the $1 / \sqrt{\cosh \theta}$ terms $^{16}$ into the Jacobian. This yields finally the decompactified expressions in the natural infinite volume normalization:

$$
\begin{aligned}
& d^{(3)}(\theta)=-\sin \frac{p L}{2} \cdot \frac{\sinh \frac{\theta}{2}}{\pi} \cdot \tilde{\Gamma}_{\frac{M L}{2 \pi}}(\theta) \cdot e^{-\frac{\theta}{2 \pi} p L} \\
& d^{(2)}(\theta)=\frac{\pi}{M L \sinh \frac{\theta}{2}} \cdot \frac{1}{\tilde{\Gamma}_{\frac{M L}{2 \pi}}(\theta)} \cdot e^{\frac{\theta}{2 \pi} p L} .
\end{aligned}
$$

It is important to note that the above expressions contain an infinite set of exponential wrapping corrections w.r.t. the size of the string $\# 1$, i.e. terms of the form $e^{-n M L}$. Later we will describe the asymptotic limit defined by neglecting these exponential corrections which turns out to be surprisingly subtle.

In appendix A we will directly formulate the continuity conditions for the decompactified SFT vertex for the massive free scalar and check that the above limit of the LSNS expression (5.21) is indeed a solution. This is important to make sure that the puzzling terms like $\sin \frac{p L}{2}$ appearing in the Neumann coefficients indeed exist directly for the decompactified vertex and do not arise from some unknown subtlety in finite volume reduction.

We can now verify that the decompactified Neumann coefficients, defined through (5.8)-(5.10) in terms of (5.21)-(5.22) satisfy the SFT vertex axioms of section 4. In the present case the symmetry (4.6) is satisfied trivially and we are left with checking the monodromy (4.7), crossing (4.3)-(4.4) and the kinematical singularity axioms (4.8).

The monodromy property is seen to be easily implemented in terms of the last factor in $(5.21)$ as $\tilde{\Gamma}_{\frac{M L}{2 \pi}}(\theta)$ is $2 \pi i$-periodic. Note that the additional signs generated by $\sinh \frac{\theta}{2}$ get canceled by signs coming from the denominators of (5.8)-(5.10).

Using the crossing property of the deformed gamma functions

$$
\tilde{\Gamma}_{\frac{M L}{2 \pi}}(\theta+i \pi) \sinh \theta \sin \frac{p L}{2}=-\frac{2 \pi^{2}}{\tilde{\Gamma}_{\frac{M L}{2 \pi}}(\theta) M L}
$$

\footnotetext{
${ }^{15}$ We choose again the factor $(-1)^{m+1}$ to be 1 .

${ }^{16}$ Since in any case we are not controlling the overall normalization here, we absorb any remaining $1 / \sqrt{M}$ factors in the normalization $\rho$.
} 
one can see the crossing relation between $d^{(3)}$ and $d^{(2)}$ is

$$
d^{(3)}(\theta \pm i \pi)=\mp i e^{ \pm i p(\theta) \frac{L}{2}} d^{(2)}(\theta) .
$$

Inserting this into (5.8)-(5.10) we get in particular the crossing properties

$$
N^{32}\left(\theta, \theta^{\prime}\right)=e^{i p\left(\theta^{\prime}\right) L / 2} N^{33}\left(\theta, \theta^{\prime}-i \pi\right) \quad N^{22}\left(\theta, \theta^{\prime}\right)=e^{i p(\theta) L / 2} N^{32}\left(\theta-i \pi, \theta^{\prime}\right) .
$$

Finally let us consider the kinematical singularity axiom (4.8) for $N^{33}\left(\theta, \theta^{\prime}\right)$. It is seen to be satisfied using the property

$$
d^{(3)}(\theta+i \pi) d^{(3)}(\theta)=-\frac{1}{2 M L}\left(1-e^{i p L}\right) .
$$

An analogous property for $N^{22}\left(\theta, \theta^{\prime}\right)$ follows from

$$
d^{(2)}(\theta+i \pi) d^{(2)}(\theta)=\frac{1}{2 M L}\left(1-e^{-i p L}\right) .
$$

Let us note some important features of the analytical properties of the (decompactified) Neumann coefficients in the complex rapidity plane. A notable feature of the functional equations (5.26) and (5.27) is that they are (almost) identical, while the explicit solutions (5.21) and (5.22) are clearly quite different. The difference lies in the location of zeroes in the physical strip. Due to the factor $\sin \frac{p L}{2}$, all the zeroes of $d^{(3)}(\theta)$ lie on the line of real $\theta$ 's, while in the case of $d^{(2)}(\theta)$, they lie on the line $\Im m(\theta)=\pi$. This directly carries over to the different location of zeroes in the Neumann coefficients $N^{r s}\left(\theta, \theta^{\prime}\right)$. Note that the physical difference between strings \#2 and \#3 is that string \#2 is accompanied by the emission of string \#1. Thus the Neumann coefficient of string \#3 vanishes exactly at the rapidities which are allowed by the asymptotic BA equations for the finite size string \#1 or by quantization around that string. We do not have currently a physical understanding of this property but expect similar features to occur for the generic interacting case.

\subsection{Asymptotic limit}

Let us now describe the asymptotic large $L$ limit of the (decompactified) Neumann coefficients or equivalently of the elementary $d^{(r)}(\theta)$ functions defined in (5.21) and (5.22). Recall that $L$ is the size of the third finite string, and the asymptotic limit is defined by neglecting all exponential $e^{-M L}$ corrections. Since this corresponds exactly to neglecting wrapping corrections, such a limit is of chief interest for the subsequent reconstruction of the finite volume SFT vertex, which we describe in section 7 , and for potential applications to OPE coefficients in $\mathcal{N}=4 \mathrm{SYM}$ theory. Incidentally, this was also exactly the relevant limit used when comparing pp-wave SFT vertex with perturbative OPE coefficients of BMN operators.

To this end, let us quote the large $M L$ asymptotics (B.10) of $\tilde{\Gamma}_{\frac{M L}{2 \pi}}(\theta)$ which follows from the properties derived in the LSNS paper [52].

$$
\tilde{\Gamma}_{\frac{M L}{2 \pi}}(\theta) \sim \sqrt{\frac{2 \pi^{2}}{M L}} \frac{e^{\frac{\theta}{2 \pi} p L}}{\sinh \frac{\theta}{2}} .
$$


It is extremely important to emphasize that the above formula holds only on an open subset $|\Im m(\theta)|<\pi$. In particular, it does not hold on the 'crossing line' $\Im m(\theta)=\pi$. A very intriguing feature of the above expression is that it has a monodromy when $\theta \rightarrow \theta+2 \pi i$ which is in apparent contradiction with the $2 \pi i$ periodicity of $\tilde{\Gamma}_{\frac{M L}{2 \pi}}(\theta)$. Of course, there is no real contradiction due to the fact that this asymptotic formula breaks down on the line $\Im m(\theta)=\pi$.

The above mentioned apparent monodromy has, however, very important consequences for the behaviour of the asymptotic Neumann coefficients. It cancels exactly the explicit monodromies in (5.21) and (5.22) and one obtains

$$
\begin{aligned}
& d^{(3)}(\theta)_{\text {asympt }}=-\sqrt{\frac{2}{M L}} \cdot \sin \frac{p L}{2} \\
& d^{(2)}(\theta)_{\text {asympt }}=\frac{1}{\sqrt{2 M L}} .
\end{aligned}
$$

This leads to the following asymptotic Neumann coefficients ${ }^{17}$ (valid for $|\Im m(\theta)|<\pi$ and $\left.\left|\Im m\left(\theta^{\prime}\right)\right|<\pi\right)$ :

$$
\begin{aligned}
& N^{33}\left(\theta, \theta^{\prime}\right)_{\text {asympt }}=-\frac{2}{M L} \frac{\sin \frac{p L}{2} \sin \frac{p^{\prime} L}{2}}{\cosh \frac{\theta-\theta^{\prime}}{2}} \\
& N^{32}\left(\theta, \theta^{\prime}\right)_{\text {asympt }}=\frac{1}{M L} \frac{\sin \frac{p L}{2}}{\sinh \frac{\theta-\theta^{\prime}}{2}} \\
& N^{22}\left(\theta, \theta^{\prime}\right)_{\text {asympt }}=\frac{1}{2 M L} \frac{1}{\cosh \frac{\theta-\theta^{\prime}}{2}} .
\end{aligned}
$$

Despite the simplicity of the above expressions, one should keep in mind that they are in fact equivalent to the all order $1 / \mu$ formulas in the pp-wave SFT vertex.

The asymptotic expressions (5.31)-(5.33) are quite intriguing. Firstly, we loose the nontrivial monodromy of the exact formulas (5.21) and (5.22) and obtain simple antiperiodic functions. We believe that this property may be necessary in the general case for solving the consistency equation (7.7) when using the decompactified formulas for constructing the finite volume SFT vertex up to wrapping corrections. Secondly, if we were to extend the above asymptotic formulas by analytical continuation to the whole complex plane, the kinematical singularity axiom and crossing property would be modified. The case of this effective asymptotic crossing is particularly intriguing as we get e.g.

$$
N^{33}\left(\theta, \theta^{\prime}-i \pi\right)_{\text {asympt }}=-2 i \sin \frac{p^{\prime} L}{2} N^{32}\left(\theta, \theta^{\prime}\right)_{\text {asympt }}
$$

which bears quite striking resemblance to the recently discovered modifications of crossing in Chern-Simons theories [54, 55].

We do not want to make here any statement about the effective asymptotic crossing and kinematical axioms in the general interacting case and leave this problem for future investigation.

\footnotetext{
${ }^{17}$ Recall that we factor out some constant normalization.
} 


\subsection{Reconstruction of $\tilde{\Gamma}_{\mu}(\theta)$ from the SFT axioms}

In the final part of this section, let us see how to reconstruct the known LSNS solution directly from solving the SFT vertex axioms of section 4. Firstly, we will see that obtaining the solution in this way is very simple, and definitely much simpler than the direct approach of [53] and [52]. Secondly, we will see that by themselves, the functional equations are not restrictive enough and one needs additional input about the analytical structure, in particular the location of zeroes, in order to fix the solution.

Let us concentrate on the $N^{33}\left(\theta, \theta^{\prime}\right)$ Neumann coefficient which is equal to our function $\mathbf{N}_{\bullet}, L\left(\theta, \theta^{\prime}\right)$ with vacuum on string \#1 (i.e. $\bullet \equiv \varnothing$ here). The functional equations in this case read

$$
\begin{aligned}
\mathbf{N}_{\varnothing, L}\left(\theta, \theta^{\prime}\right) & =\mathbf{N}_{\varnothing, L}\left(\theta^{\prime}, \theta\right) \\
\mathbf{N}_{\varnothing, L}\left(\theta+2 \pi i, \theta^{\prime}\right) & =e^{-i p L} \mathbf{N}_{\varnothing, L}\left(\theta, \theta^{\prime}\right) \\
\mathbf{N}_{\varnothing, L}(\theta+i \pi+\varepsilon, \theta) & =\frac{i}{\varepsilon}\left(1-e^{i p L}\right) \mathbf{N}_{\varnothing, L}+\mathcal{O}\left(\varepsilon^{0}\right)
\end{aligned}
$$

and we take $\mathbf{N}_{\varnothing, L}$ with no arguments to be equal to 1 (i.e. we normalize the answer w.r.t. taking the amplitude with vacuum on all three strings). We further assume that the large real $\theta$ asymptotics of the solution is bounded.

It is convenient to solve first the monodromy axiom by factoring out

$$
e^{-\frac{\theta}{2 \pi} p L-\frac{\theta^{\prime}}{2 \pi} p^{\prime} L}
$$

from $\mathbf{N}_{\varnothing, L}\left(\theta, \theta^{\prime}\right) \equiv N^{33}\left(\theta, \theta^{\prime}\right)$. Also we may implement the kinematical singularity by introducing a denominator $e^{\theta}+e^{\theta^{\prime}}$, i.e.

$$
N^{33}\left(\theta, \theta^{\prime}\right)=\frac{e^{-\frac{\theta}{2 \pi} p L-\frac{\theta^{\prime}}{2 \pi} p^{\prime} L}}{e^{\theta}+e^{\theta^{\prime}}} Q\left(\theta, \theta^{\prime}\right) .
$$

The kinematical singularity axiom implies the following functional equation for $Q\left(\theta, \theta^{\prime}\right)$ :

$$
Q(\theta+i \pi, \theta) i e^{-\theta}=e^{-i p \frac{L}{2}}-e^{i p \frac{L}{2}} .
$$

Let us introduce a simple factorizable ansatz for $Q\left(\theta, \theta^{\prime}\right)$ :

$$
Q\left(\theta, \theta^{\prime}\right)=\frac{2 h(\theta)}{1+e^{-\theta}} \frac{2 h\left(\theta^{\prime}\right)}{1+e^{-\theta^{\prime}}}
$$

Then we have

$$
h(\theta) h(\theta+i \pi)=-\sinh \theta \sin \frac{p L}{2} .
$$

We recover thus the functional equation for $1 / \tilde{\Gamma}_{\frac{M L}{2 \pi}}(\theta)$ up to a normalization factor. It is nevertheless instructive to try to solve this directly in order to rederive the special function $\tilde{\Gamma}_{\mu}$ and also to understand its space of solutions. In particular, we can verify directly that

$$
\frac{1}{\tilde{\Gamma}_{\frac{M L}{2 \pi}}(\theta+i \pi)} \propto \sinh \theta \sin \frac{p L}{2} \cdot \tilde{\Gamma}_{\frac{M L}{2 \pi}}(\theta)
$$

is also a solution (up to an appropriate overall constant). 
We can rewrite the functional equation (5.42) as

$$
h(p) h(-p)=-p \sin \frac{p L}{2}
$$

and expand the sine into an infinite product $\sin \pi z=\pi z \prod_{n=1}^{\infty}\left(1-\frac{z^{2}}{n^{2}}\right)$. We can find a solution in terms of a product of elementary solutions solving

$$
f(p) f(-p)=\left(1-\frac{p^{2} L^{2}}{4 \pi^{2} n^{2}}\right) .
$$

The right hand side has two zeroes and a-priori we are free to distribute them either into the first or second factor on the left hand side or into both of them. This corresponds to the choice whether the zeroes lie on the line $\Im m(\theta)=0$ or $\Im m(\theta)=\pi$. From the exact LSNS solution for $N^{33}\left(\theta, \theta^{\prime}\right)$ discussed in section 5.2, we see that both zeroes should be on the real line. The simplest and most obvious solution $f(\theta)=1-p L /(2 \pi n)$ does not provide the right location of zeroes as it leads to zeroes lying on both of the two lines and we need a slightly more involved factorization:

$$
\left(\sqrt{M^{2}+\frac{4 \pi^{2} n^{2}}{L^{2}}}-E(\theta)\right)\left(\sqrt{M^{2}+\frac{4 \pi^{2} n^{2}}{L^{2}}}-E(\theta+i \pi)\right)=\frac{4 \pi^{2} n^{2}}{L^{2}}-p^{2}
$$

where $E(\theta)=M \cosh \theta$. We are now free to include either of the two factors into $f(\theta)$ and consequently into $h(\theta)$. If we choose the right hand factors for all $n$, the solution will not have zeroes on the real line. The consistent choice of the left hand factor would conversely ensure that all the zeroes lie on the real axis - this choice will lead to the second solution (5.43) relevant for $N^{33}\left(\theta, \theta^{\prime}\right)$. However, we could have made different choices for any $n$ constructing many (nonphysical) solutions of (5.42). This shows that the assumptions on the location of zeroes are of crucial importance.

Introducing appropriate exponential factors for convergence leads to the infinite product representation of the $\tilde{\Gamma}_{\mu}(\theta)$ following from the formulas in appendix B.

\section{Axioms for the nondiagonal case}

In this section we formulate axioms for the SFT vertex in the case when the integrable worldsheet theory contain particles of different types: additionally to the rapidity, $\theta$, the particles are characterized also by their particle type: $i$. In general, the scattering matrix is non-diagonal, but due to integrability, the multiparticle scatterings factorize into two particle scatterings, and the S-matrix satisfies crossing symmetry:

$$
S_{i j}^{k l}\left(\theta_{1}, \theta_{2}\right)=C^{k \bar{k}} S_{j \bar{k}}^{l \bar{i}}\left(\theta_{2}, \theta_{1}-i \pi\right) C_{\bar{i} i}=C^{l \bar{l}} S_{\overline{l i}}^{\bar{j} k}\left(\theta_{2}+i \pi, \theta_{1}\right) C_{\bar{j} j}
$$

where $C_{i j}$ is the charge conjugation matrix and its inverse is $C^{j k}: C_{i j} C^{j k}=\delta_{i}^{k}$. The crossing transformation connects anti-particles in the initial state to particles in the final state and vica versa. The graphical representation of the crossing symmetry of the scattering matrix is demonstrated on figure 11 . 


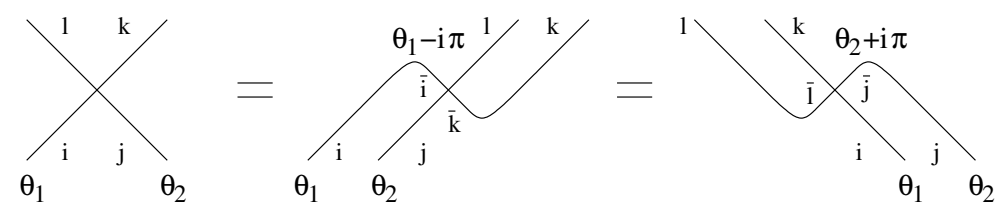

Figure 11. Crossing transformation of the scattering matrix.

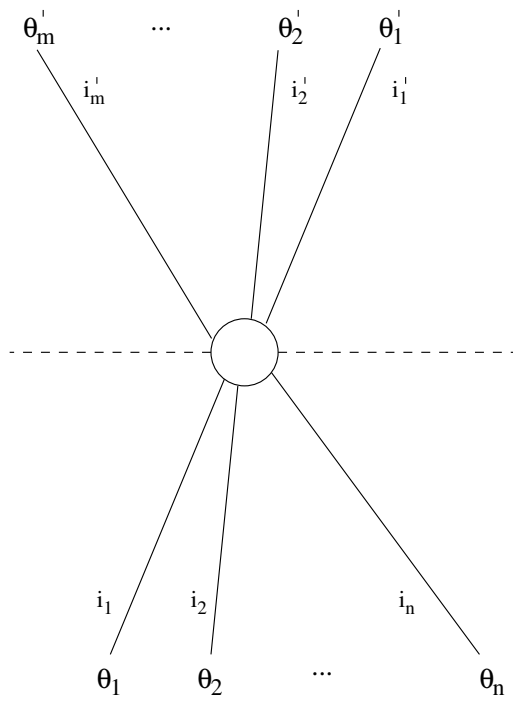

Figure 12. SFT vertex in the generic case. The initial state contains particles with rapidities $\theta_{1}, \ldots, \theta_{n}$ and particle content $i_{1}, \ldots, i_{n}$, while the final state, has rapidities $\theta_{1}^{\prime}, \ldots, \theta_{m}^{\prime}$ and particle content $i_{1}^{\prime}, \ldots, i_{m}^{\prime}$.

The decompactified SFT vertex, additionally to the rapidities, depends also on the types of the particles which we denote as

$$
N_{\bullet}^{3 \mid 2}\left(\theta_{1}, \ldots, \theta_{n} \mid \theta_{1}^{\prime}, \ldots, \theta_{m}^{\prime}\right)_{i_{1}, \ldots, i_{n}}^{i_{1}^{\prime}, \ldots, i_{m}^{\prime}}
$$

where $\theta_{1}, \ldots, \theta_{n}$ are the rapidities of the initial state in domain \#3 with particle content $i_{1}, \ldots, i_{n}$, while the final state, in domain $\# 2$, has rapidities $\theta_{1}^{\prime}, \ldots, \theta_{m}^{\prime}$ and particle content $i_{1}^{\prime}, \ldots, i_{m}^{\prime}$. The placement of the indices and their orderings reflect the geometry of the amplitude as shown on figure 12 .

By keeping the previous convention we distribute the space discontinuity equally on the border of domains \#3 and \#2. The space deficiency has no effect on the particles type so the generalized crossing relations take the form

$$
\begin{aligned}
& N_{\bullet}^{3 \mid 2}\left(\theta_{1}, \ldots, \theta_{n} \mid \theta_{1}^{\prime}, \ldots, \theta_{m}^{\prime}\right)_{i_{1}, \ldots, i_{n}}^{i_{1}^{\prime}, \ldots, i_{m}^{\prime}}=e^{i p\left(\theta_{1}^{\prime}\right) L / 2} N_{\bullet, L}^{3 \mid 2}\left(\theta_{1}, \ldots, \theta_{n}, \theta_{1}^{\prime}-i \pi \mid \theta_{2}^{\prime}, \ldots, \theta_{m}^{\prime}\right)_{i_{1}, \ldots, i_{n}, \bar{j}}^{i_{2}^{\prime}, \ldots, i_{m}^{\prime}} C^{\bar{j} i_{1}^{\prime}} \\
& N_{\bullet, L}^{3 \mid 2}\left(\theta_{1}, \ldots, \theta_{n} \mid \theta_{1}^{\prime}, \ldots, \theta_{m}^{\prime}\right)_{i_{1}, \ldots, i_{n}}^{i_{1}^{\prime}, \ldots, i_{m}^{\prime}}=e^{-i p\left(\theta_{m}^{\prime}\right) L / 2} N_{\bullet}^{3 \mid 2}\left(\theta_{m}^{\prime}-i \pi, \theta_{1}, \ldots, \theta_{n} \mid \theta_{1}^{\prime}, \ldots, \theta_{m-1}^{\prime}\right)_{\bar{j}, i_{1}, \ldots, i_{n}}^{i_{1}^{\prime}, \ldots, i_{m-1}^{\prime}} C^{\bar{j} i_{m}^{\prime}}
\end{aligned}
$$

Graphically they can be represented as we show on figure 13. These crossing relations are valid if the incoming and outgoing particle states have no overlaps, as otherwise singularities can appear. We explain later these disconnected terms, which are related to 

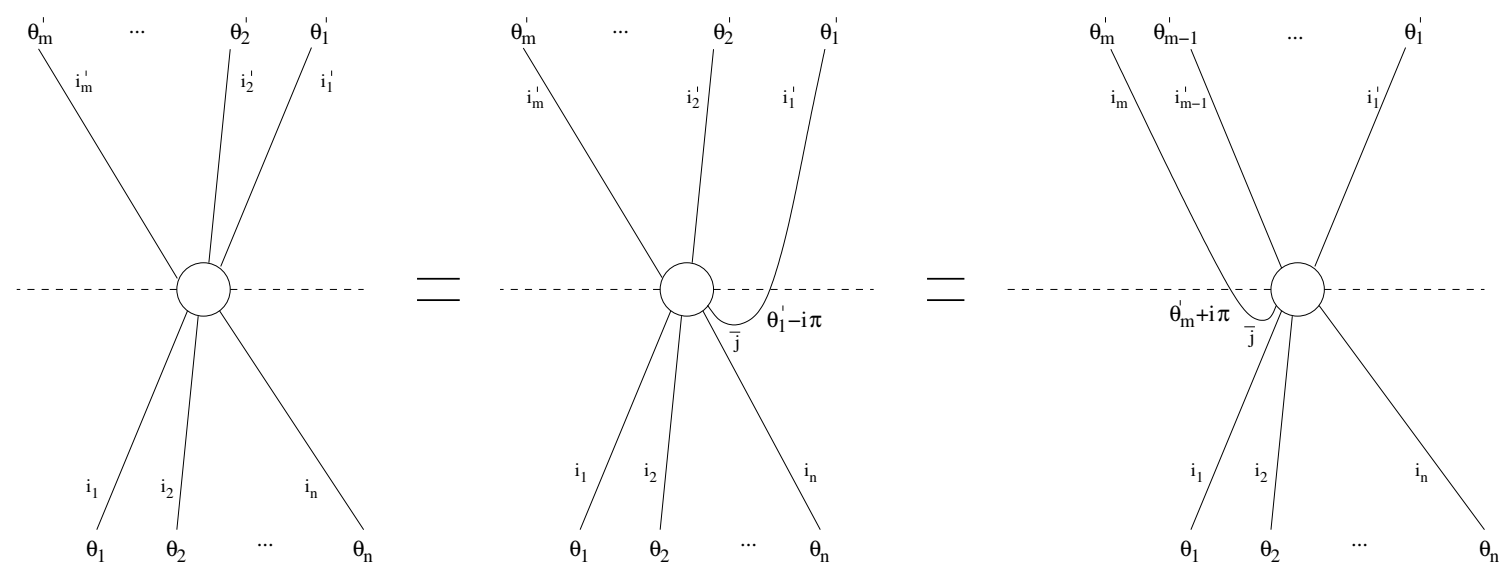

Figure 13. Graphical representation of the crossing transformation in the nondiagonal case. Crossing a particle from outgoing to incoming comes with a charge conjugation matrix, which replaces the particle with its antiparticle.

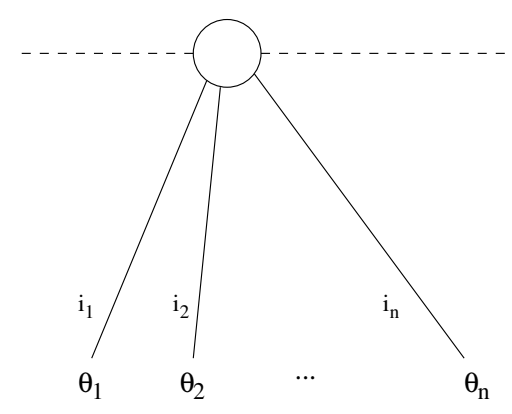

Figure 14. The elementary SFT vertex in the generic case.

amplitudes with less particles. By crossing all particles into the initial state we can define the elementary SFT vertex

$$
N_{\bullet, L}\left(\theta_{1}, \ldots, \theta_{n}\right)_{i_{1}, \ldots, i_{n}}=N_{\bullet, L}^{3 \mid 2}\left(\theta_{1}, \ldots, \theta_{n} \mid \varnothing\right)_{i_{1}, \ldots, i_{n}}
$$

which we represent graphically on figure 14 .

This elementary SFT vertex satisfies several axioms. The permutation axiom expresses that exchanging two particles comes with an S-matrix factor:

$$
\begin{aligned}
& N_{\bullet}, L\left(\theta_{1}, \ldots, \theta_{j}, \theta_{j+1}, \ldots, \theta_{n}\right)_{i_{1}, \ldots i_{j}, i_{j+1}, \ldots, i_{n}} \\
& \quad=S_{i_{j} i_{j+1}}^{k l}\left(\theta_{j}, \theta_{j+1}\right) N_{\bullet, L}\left(\theta_{1}, \ldots, \theta_{j+1}, \theta_{j}, \ldots, \theta_{n}\right)_{i_{1}, \ldots l, k, \ldots, i_{n}}
\end{aligned}
$$

Graphically it takes the form shown on figure 15. By crossing the leftmost incoming particle to an outgoing antiparticle and crossing back again to the rightmost particle we obtain the monodromy property

$$
N_{\bullet, L}\left(\theta_{1}, \ldots, \theta_{n}\right)_{i_{1}, \ldots, i_{n}}=e^{-i p\left(\theta_{1}\right) L} N_{\bullet} L\left(\theta_{2}, \ldots, \theta_{n}, \theta_{1}-2 i \pi\right)_{i_{2}, \ldots, i_{n}, i_{1}}
$$

which is shown on figure 16 . 

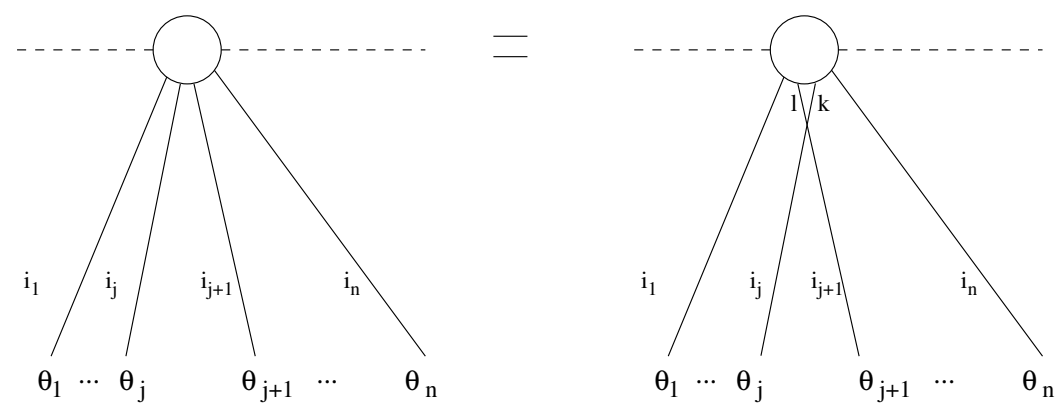

Figure 15. Permutation axiom for the SFT vertex. Exchanging two neighbouring particles introduces an S-matrix factor.
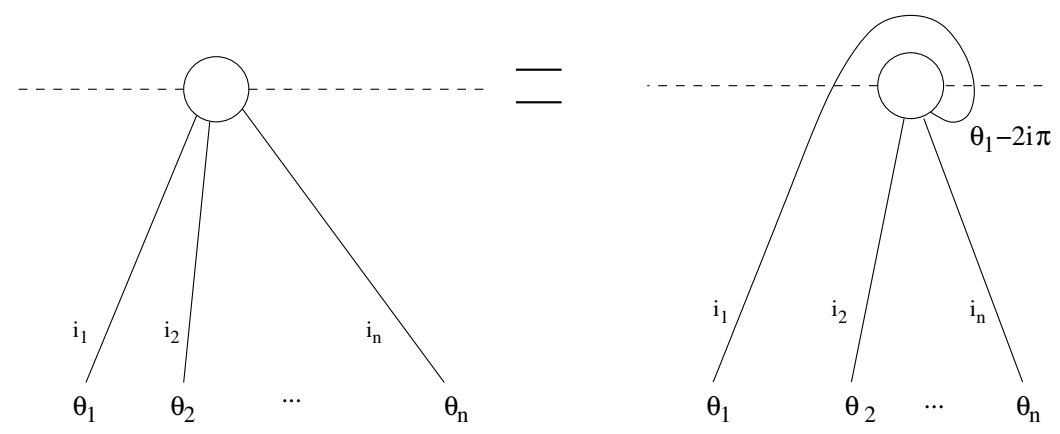

Figure 16. Monodromy property for the generic SFT vertex.

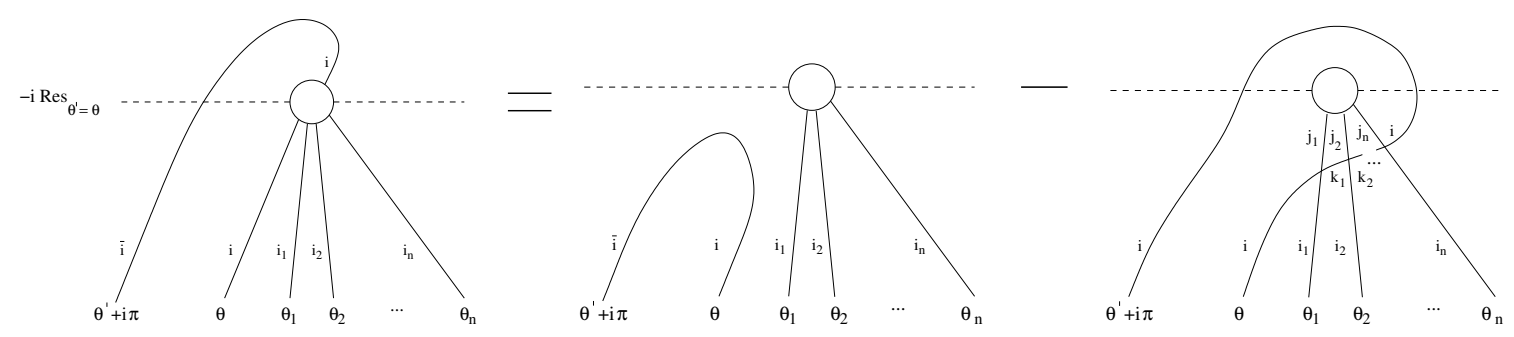

Figure 17. Kinematical singularity axiom for the generic SFT vertex.

The crossing relation is valid if none of the incoming particles coincides with any of the outgoing particles. Otherwise, the amplitude is singular, but the residue of the pole is related to an onshell propagation of the particle passing the reduced amplitude on both sides:

$$
\begin{aligned}
-i \operatorname{Res}_{\theta^{\prime}=\theta} N_{\bullet}, L & \left(\theta^{\prime}+i \pi, \theta, \theta_{1}, \ldots, \theta_{n}\right)_{\bar{i}, i, i_{1}, \ldots, i_{n}} \\
& =\left(\delta_{i_{1} \ldots i_{n}}^{j_{1} \ldots j_{n}}-e^{i p(\theta) L} S_{i i_{1}}^{k_{1} j_{1}}\left(\theta, \theta_{1}\right) \ldots S_{k_{n-1} i_{n}}^{i j_{n}}\left(\theta, \theta_{1}\right)\right) \times N_{\bullet, L}\left(\theta_{1}, \ldots, \theta_{n}\right)_{j_{1}, \ldots, j_{n}} .
\end{aligned}
$$

This axiom is called the kinematical singularity axioms, which connects the amplitude with $n+2$ particles to an amplitude with $n$ particles. This process is indicated on figure 17 Singularities of the SFT vertex always correspond to some kinematically allowed onshell propagation of the particles. If, for example, two particles with labels $i$ and $j$, with rapidities $\theta-i \nu$ and $\theta+i \nu$, can form a boundstate of type $k$ with rapidity $\theta$, then the SFT 

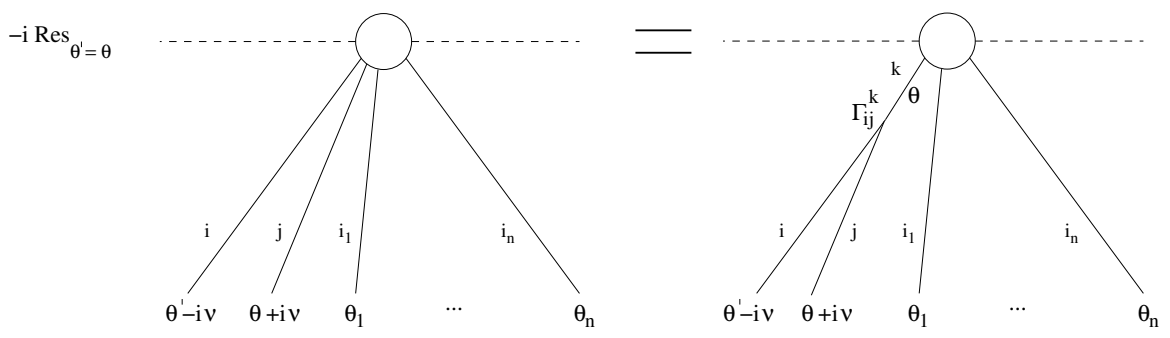

Figure 18. Dynamical singularity axiom for the SFT vertex.

vertex is singular and its residue is related to the SFT vertex of the boundstate as

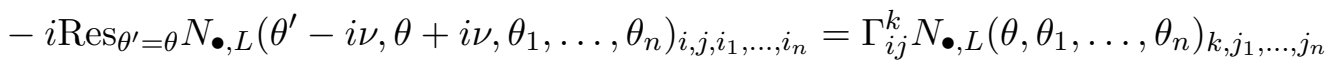

where $\Gamma_{i j}^{k}$ is the strength of the coupling, which is related to the residue of the pole in the scattering matrix

$$
-i \operatorname{Res}_{\theta^{\prime}=\theta} S_{i j}^{k l}\left(\theta^{\prime}+i \nu, \theta-i \nu\right)=\Gamma_{i j}^{m} \Gamma_{m}^{k l} .
$$

This singularity axiom is called the dynamical singularity axiom and is represented graphically on figure 18. Generally, for any onshell propagation we have a singularity of the SFT vertex. This is similar to how the singularities of the scattering matrix can be explained by Coleman-Thun diagrams.

In formulating the nondiagonal SFT vertex axioms we used rapidity parametrizations $\theta$, with crossing transformations $\theta \rightarrow \theta \pm i \pi$, but we did not assume any relativistic invariance for the scattering matrix. The generalizations of these formulas for the AdS/CFT integrable model can be obtained by using its rapidity parametrizations $\theta \rightarrow z$ and its crossing transformations $z \rightarrow \pm \omega_{2}$. These axioms are very similar to the form factor axioms for world-sheet operators, [42], except the factor $e^{i p L}$ appearing in the monodromy/periodicity equations.

\section{The program for the finite volume string vertex}

Let us now formulate our program for the general finite volume string field theory vertex up to wrapping corrections. As explained before, we do not expect a-priori an exponential form of the vertex expressed in terms of some generalized Neumann coefficients ${ }^{18}$ so what we are after is a general amplitude with any prescribed multiparticle state on each string. In the limit that we are considering, i.e. neglecting wrapping corrections, these states will be parametrized by momenta solving Asymptotic Bethe Ansatz equations for each string individually. ${ }^{19}$ We thus want to determine

$$
\mathbf{N}_{L_{3} \mid L_{2} ; L_{1}}^{3 \mid 2 ; 1}\left(\left\{p_{i}^{(3)}\right\} \mid\left\{p_{j}^{(2)}\right\} ;\left\{p_{k}^{(1)}\right\}\right)
$$

where we explicitly indicated the sizes of the respective strings.

\footnotetext{
${ }^{18}$ Although a-posteriori a generalization might exist in analogy to similar structures for boundary states in integrable relativistic QFT's [27].

${ }^{19}$ And of course the standard additional Bethe roots associated to nesting. In the following, for brevity, we just explicitly indicate only the momenta.
} 
Our program consists of first solving the SFT functional equations derived in section 4 for the two distinct decompactified versions of (7.1), namely

$$
\mathbf{N}_{\left\{p_{k}^{(1)}\right\}, L_{1}}^{3 \mid 2}\left(\left\{p_{i}^{(3)}\right\} \mid\left\{p_{j}^{(2)}\right\}\right)
$$

and

$$
\mathbf{N}_{\left\{p_{j}^{(2)}\right\}, L_{2}}^{3 \mid 1}\left(\left\{p_{i}^{(3)}\right\} \mid\left\{p_{k}^{(1)}\right\}\right) .
$$

It is important to note that in (7.2), the momenta $\left\{p_{i}^{(3)}\right\}$ and $\left\{p_{j}^{(2)}\right\}$ are assumed to be unconstrained and that the relevant SFT axioms involve explicitly only these momenta. There is no dependence in these axioms on the $\left\{p_{k}^{(1)}\right\}$, however there is certainly a huge freedom in the choice of a particular solution which may depend on the $\left\{p_{k}^{(1)}\right\}$. This is in direct analogy to the case of form factors where the axioms do not depend on the particular choice of local operator. They allow, however, for many solutions associated to different choices of local operators. In the SFT vertex case we will soon show how to strongly constrain this dependence.

Similarly, in the case of (7.3), the axioms involve explicitly only momenta $\left\{p_{i}^{(3)}\right\}$ and $\left\{p_{k}^{(1)}\right\}$, and the particular solutions should a-priori depend on the $\left\{p_{j}^{(2)}\right\}$ this time.

Our main point is now that performing finite volume reduction from (7.2) and (7.3) should yield exactly the same expression, which will be the original quantity of interest (7.1).

Since we are working only up to wrapping corrections, we should neglect ${ }^{20}$ wrapping corrections (w.r.t. respectively $L_{1}$ and $L_{2}$ ) in the decompactified solutions (7.2) and (7.3). We denote the resulting asymptotic solutions with a subscript asympt e.g.

$$
\mathbf{N}_{\left\{p_{k}^{3 \mid 2}\right\}, L_{1}}^{(2)}\left(\left\{p_{i}^{(3)}\right\} \mid\left\{p_{j}^{(2)}\right\}\right)_{\text {asympt }} .
$$

This is the direct counterpart of the asymptotic formulas of section 5.3 which were given for the case of the massive free boson (the pp-wave case). Note that these expressions will typically still have some oscillatory $L_{1,2}$ dependence in factors like $\sin \frac{p L_{1}}{2}$. Now again up to wrapping corrections, the finite volume reduction should just amount to multiplying by the same factors as for finite volume form factors. We thus get (up to wrapping corrections)

$$
\mathbf{N}_{L_{3} \mid L_{2} ; L_{1}}^{3 \mid 2 ; 1}\left(\left\{p_{i}^{(3)}\right\} \mid\left\{p_{j}^{(2)}\right\} ;\left\{p_{k}^{(1)}\right\}\right)=\frac{1}{\sqrt{\tilde{\rho}_{3} \tilde{\rho}_{2}}} \mathbf{N}_{\left\{p_{k}^{(1)}\right\}, L_{1}}^{3 \mid 2}\left(\left\{p_{i}^{(3)}\right\} \mid\left\{p_{j}^{(2)}\right\}\right)_{\text {asympt }}
$$

where $\tilde{\rho}$ is the same factor as in (2.8), involving the Gaudin norm together with a product of S-matrices. It is important to note, that we could have equally well obtained the same expression from the second decompactification

$$
\mathbf{N}_{L_{3} \mid L_{2} ; L_{1}}^{3 \mid 2 ; 1}\left(\left\{p_{i}^{(3)}\right\} \mid\left\{p_{j}^{(2)}\right\} ;\left\{p_{k}^{(1)}\right\}\right)=\frac{1}{\sqrt{\tilde{\rho}_{3} \tilde{\rho}_{1}}} \mathbf{N}_{\left\{p_{j}^{(2)}\right\}, L_{2}}^{3 \mid 1}\left(\left\{p_{i}^{(3)}\right\} \mid\left\{p_{k}^{(1)}\right\}\right)_{\text {asympt }}
$$

\footnotetext{
${ }^{20}$ It is possible that keeping the full decompactified solution in the following steps will yield lot of information on some wrapping corrections for the OPE coefficients. However then the matching of the finite volume reductions might require some care. We leave this possibility for future investigation.
} 

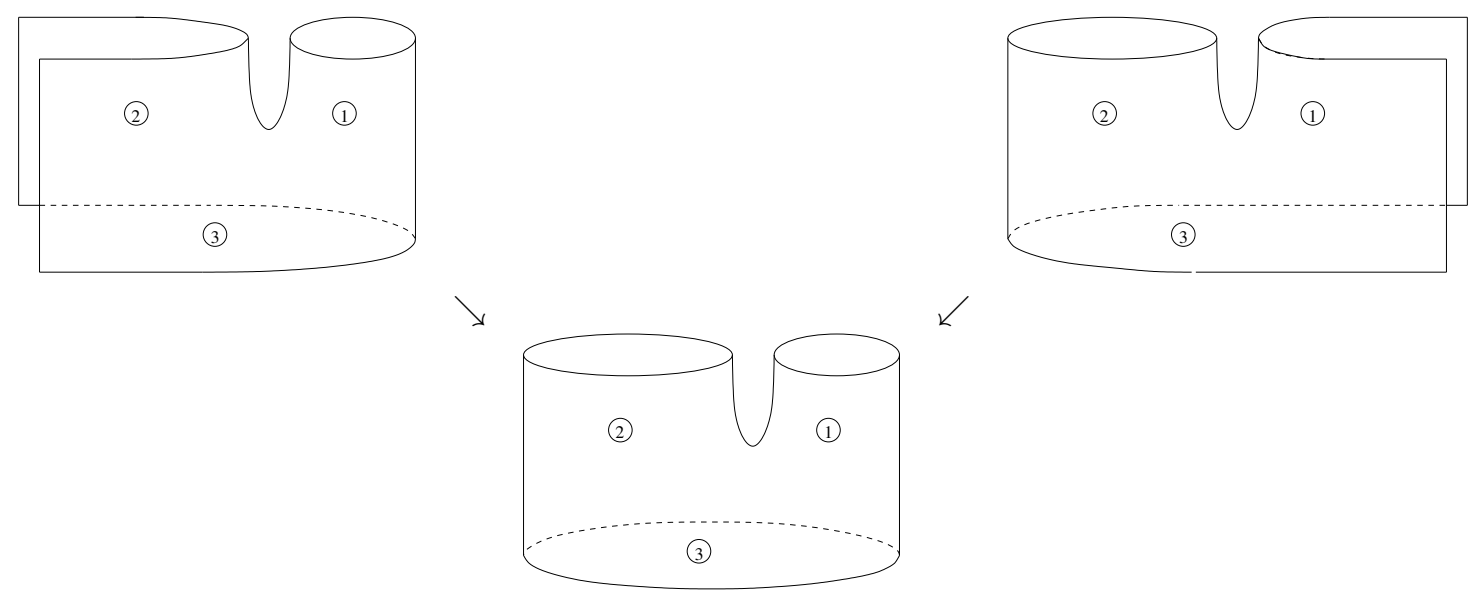

Figure 19. The program for obtaining the finite volume string field theory vertex up to wrapping corrections.

The consistency of these two expressions provides for us the key final equation

$$
\frac{1}{\sqrt{\tilde{\rho}_{2}}} \mathbf{N}_{\left\{p_{k}^{(1)}\right\}, L_{1}}^{3 \mid 2}\left(\left\{p_{i}^{(3)}\right\} \mid\left\{p_{j}^{(2)}\right\}\right)_{\text {asympt }}=\frac{1}{\sqrt{\tilde{\rho}_{1}}} \mathbf{N}_{\left\{p_{j}^{(2)}\right\}, L_{2}}^{3 \mid 1}\left(\left\{p_{i}^{(3)}\right\} \mid\left\{p_{k}^{(1)}\right\}\right)_{\text {asympt }}
$$

which should very strongly constrain the $\left\{p_{k}^{(1)}\right\}$-dependent choice of particular solution of the SFT axioms for (7.2) and the $\left\{p_{j}^{(2)}\right\}$-dependent choice of solution of the SFT axioms for (7.3). The resulting expression yields the final finite volume amplitude (7.1). We illustrate pictorially this strategy in figure 19 .

\subsection{The program for the simplest plane wave SFT vertex}

Let us see how to implement the program above for the simplest pp-wave SFT vertex, namely for the asymptotic value of $N^{33}\left(\theta, \theta^{\prime}\right)$, which is denoted by

$$
N_{L_{3} \mid L_{2}, L_{1}}^{3 \mid 2,1}\left(p, p^{\prime} \mid \varnothing ; \varnothing\right)_{\text {asympt }} .
$$

We use the previously calculated results but put into the context of the general program.

As a first step we decompactify $L_{2}$ and $L_{3}$ and determine $N_{\varnothing \mid L_{1}}^{3 \mid 2}\left(\theta, \theta^{\prime} \mid \varnothing\right)$ from our axioms as

$$
N_{\varnothing, L_{1}}^{3 \mid 2}\left(\theta, \theta^{\prime} \mid \varnothing\right)=n\left(L_{1}\right) \sin \frac{p L_{1}}{2} \sin \frac{p^{\prime} L_{1}}{2} \frac{\sinh \frac{\theta}{2} \sinh \frac{\theta^{\prime}}{2}}{\pi^{2} \cosh \frac{1}{2}\left(\theta-\theta^{\prime}\right)} \cdot \tilde{\Gamma}_{\frac{M L_{1}}{2 \pi}}(\theta) \tilde{\Gamma}_{\frac{M L_{1}}{2 \pi}}\left(\theta^{\prime}\right) \cdot e^{-\frac{L_{1}}{2 \pi}\left(p \theta+p^{\prime} \theta^{\prime}\right)}
$$

where $n\left(L_{1}\right)$ is a normalization factor depending on the state in the finite string $\# 1$.

In the next step we take the $L_{1} \rightarrow \infty$ limit and neglect all exponentially small, $e^{-m L_{1}}$ corrections:

$$
N_{\varnothing, L_{1}}^{3 \mid 2}\left(\theta, \theta^{\prime} \mid \varnothing\right)_{\text {asympt }}=n\left(L_{1}\right) \sin \frac{p L_{1}}{2} \sin \frac{p^{\prime} L_{1}}{2} \frac{1}{\cosh \frac{1}{2}\left(\theta-\theta^{\prime}\right)} .
$$

Now we repeat the same calculations for $L_{2}$. We decompactify $L_{1}$ and $L_{3}$ and solve the functional equations for $N_{\varnothing \mid L_{2}}^{3 \mid 1}\left(\theta, \theta^{\prime} \mid \varnothing\right)$. The result is the same as (7.9) but $L_{1}$ is exchanged 
with $L_{2}$. After taking the $L_{2} \rightarrow \infty$ limit and neglecting wrapping corrections we obtain the asymptotic form:

$$
N_{\varnothing, L_{2}}^{3 \mid 1}\left(\theta, \theta^{\prime} \mid \varnothing\right)_{\text {asympt }}=n\left(L_{2}\right) \sin \frac{p L_{2}}{2} \sin \frac{p^{\prime} L_{2}}{2} \frac{1}{\cosh \frac{1}{2}\left(\theta-\theta^{\prime}\right)} .
$$

Using that there are no particles in strings $\# 1$ and $\# 2$, thus $\rho_{1}=\rho_{2}=1$, we demand that

$$
n\left(L_{1}\right) \sin \frac{p L_{1}}{2} \sin \frac{p^{\prime} L_{1}}{2} \frac{1}{\cosh \frac{1}{2}\left(\theta-\theta^{\prime}\right)}=n\left(L_{2}\right) \sin \frac{p L_{2}}{2} \sin \frac{p^{\prime} L_{2}}{2} \frac{1}{\cosh \frac{1}{2}\left(\theta-\theta^{\prime}\right)} .
$$

Recall that $L_{3}=L_{1}+L_{2}$ and that both $p$ and $p^{\prime}$ satisfy the asymptotic BA equations $e^{i p L_{3}}=1=e^{i p^{\prime} L_{3}}$ together with the level matching condition ${ }^{21} p=-p^{\prime}$. This implies that

$$
\sin \frac{p L_{1}}{2} \sin \frac{p^{\prime} L_{1}}{2}=\sin \frac{p\left(L_{3}-L_{2}\right)}{2} \sin \frac{p^{\prime}\left(L_{3}-L_{2}\right)}{2}=\sin \frac{p L_{2}}{2} \sin \frac{p^{\prime} L_{2}}{2}
$$

and we are forced to take $n\left(L_{1}\right)=n\left(L_{2}\right)=n$. The asymptotic SFT vertex is then

$$
N_{L_{3} \mid L_{2}, L_{1}}^{3 \mid 2,1}\left(p, p^{\prime} \mid \varnothing ; \varnothing\right)_{\mathrm{asympt}}=\frac{1}{\sqrt{\rho_{1} \rho_{3}}} N_{\varnothing, L_{1}}^{3 \mid 2}\left(\theta, \theta^{\prime} \mid \varnothing\right)_{\mathrm{asympt}}=\frac{n^{\prime}}{\sqrt{\cosh \theta \cosh \theta^{\prime}}} \frac{\sin \frac{p L_{1}}{2} \sin \frac{p^{\prime} L_{1}}{2}}{\cosh \frac{1}{2}\left(\theta-\theta^{\prime}\right)}
$$

where we used that $\rho_{1}=1 ; \rho_{3}=M^{2} L_{1}^{2} \cosh \theta \cosh \theta^{\prime}$ and absorved $1 / M L_{1}$ into the normalization $n^{\prime}$. Clearly this answer agrees with the asymptotic form of the relevant pp-wave finite volume Neumann coefficient.

\section{Weak coupling cross-checks with OPE coefficients}

In this section we comment on how the kinematical singularity axiom is satisfied at weak coupling directly for the OPE coefficient. More details can be found in appendix C. We investigate the 3-point functions in the $s u(1 \mid 1)$ and $s u(2)$ sectors up to 1-loop based on the available explicit results obtained from direct gauge theory calculations in $[57,58]$. As we do not know the exact relation between the 3-point functions and the SFT vertex we neglect the proper infinite volume normalization factors and check the axioms only up to some proportionality factor.

Even this comparision is not straightforward in two respects: firstly, at weak coupling the kinematical domains of the crossed amplitudes are infinitely far from each other and no longer connected analytically. Secondly, the weak coupling results were calculated for operators of finite sizes $\left(L_{i}\right)$, however our axioms are valid when two volumes ( $L_{3}$ and $L_{2}$ ) were sent to infinity by keeping the third volume $\left(L_{1}=L_{3}-L_{2}\right)$ finite.

We can circumvent these problems: first, by formulating the kinematical singularity axioms directly for the crossed process, that is, when we have the same type of particles in the initial state (operator $\mathcal{O}_{3}$ ) and in the final state (operator $\mathcal{O}_{2}$ ), and second, by taking a careful limit of the finite volume formulas. To spell out the details let us denote the

\footnotetext{
${ }^{21}$ Here we used the level matching condition to get rid of the extra factor $(-1)^{m}$, with $m$ being the BA quantization number.
} 
momenta of the initial state by $p_{j}^{(3)}$ and those of the final state by $p_{i}^{(2)}$. The reformulation of the kinematical singularity axiom into this setting means that the OPE coefficient $C_{123}(\{p\})$ must have a pole whenever $p_{n}^{(3)}=p_{m}^{(2)}$ with the residue

$$
-i \operatorname{Res} C_{123}(\{p\}) \propto\left(1-e^{i p_{n}^{(3)} L_{1}} \prod_{j}^{N_{2}} S\left(p_{j}^{(2)}, p_{n}^{(3)}\right) \prod_{k}^{N_{3}} S\left(p_{n}^{(3)}, p_{k}^{(3)}\right)\right) \times C_{123}\left(\{p\} \backslash\left\{p_{n}^{(3)}, p_{m}^{(2)}\right\}\right)
$$

where $S\left(p_{1}, p_{2}\right)$ is the scattering matrix, which we choose to be diagonal. This motivates us to analyze the $s u(2)$ and $s u(1 \mid 1)$ closed diagonal subsectors of the theory. Observe that by sending $L_{3}$ and $L_{2}$ to infinity we went off-shell with the momenta, which is crucial to have the singularity. For finite $L_{3}$ and $L_{2}$ volumes the BA equations

$$
e^{i p_{n}^{(3)} L_{3}} \prod_{j: j \neq n} S\left(p_{n}^{(3)}, p_{j}^{(3)}\right)=1 ; \quad e^{i p_{m}^{(2)} L_{2}} \prod_{k: k \neq m} S\left(p_{m}^{(2)}, p_{k}^{(2)}\right)=1
$$

kill the singularity. Let us emphasize that the power of the clear analytical properties shows up only in the infinite volume limit.

In the following we explain how to extract the infinite volume limit of the 3-point functions in the $s u(1 \mid 1)$ and $s u(2)$ cases. In both cases the explicit volume $\left(L_{2}\right.$ or $\left.L_{3}\right)$ dependence comes from a term of the form

$$
1-e^{-i p_{n}^{(3)} L_{2}} \prod_{j: j \neq n}^{N_{2}} S\left(p_{j}^{(2)}, p_{n}^{(3)}\right)
$$

which prevents us to take the $L_{2} \rightarrow \infty$ limit. The idea is to use the Bethe Ansatz equation for $p_{n}^{(3)}$ to replace the expression above with

$$
1-e^{i p_{n}^{(3)} L_{1}} \prod_{j}^{N_{2}} S\left(p_{j}^{(2)}, p_{n}^{(3)}\right) \prod_{k}^{N_{3}} S\left(p_{n}^{(3)}, p_{k}^{(3)}\right)
$$

where $L_{1}=L_{3}-L_{2}$ is kept finite in the required limit, which now exists. Using this procedure and some renormalization of the states we obtained the infinite volume 3-point function in the two sectors as follows.

\subsection{The $s u(1 \mid 1)$ sector}

The infinite volume limit of the OPE coefficient ${ }^{22}$ in the $s u(1 \mid 1)$ sector up to 1-loop takes the form:

$$
C_{123}(\{p\}) \propto \frac{\prod_{r=1}^{2} \prod_{i<j}^{N_{r}} f\left(p_{i}^{(r)}, p_{j}^{(r)}\right)}{\prod_{i}^{N_{3}} \prod_{j}^{N_{2}} f\left(p_{i}^{(3)}, p_{j}^{(2)}\right)} \prod_{l}^{N_{3}}\left(1-e^{i p_{l}^{(3)} L_{1}} \prod_{i}^{N_{2}} S\left(p_{i}^{(2)}, p_{l}^{(3)}\right) \prod_{j}^{N_{3}} S\left(p_{l}^{(3)}, p_{j}^{(3)}\right)\right)
$$

where the momenta $p^{(3)}$ and $p^{(2)}$ no longer satisfy any quantization condition, $S$ denotes the scattering matrix in the $s u(1 \mid 1)$ sector and $f$ is a known function, (displayed in appendix $\mathrm{C}$ ),

\footnotetext{
${ }^{22}$ More precisely of its complex conjugate w.r.t. the form written in [57].
} 
whose explicit form is not relevant for us now, except that it vanishes at coinciding arguments.

In order to check the kinematical residue axiom at $p_{k}^{(3)}=p_{j}^{(2)}$ we calculate

$$
\frac{-i \operatorname{Res}_{p_{k}^{(3)}=p_{j}^{(2)}} C_{123}(\{p\})}{C_{123}\left(\{p\} \backslash\left\{p_{k}^{(3)}, p_{j}^{(2)}\right\}\right)} .
$$

The prefactor containing $f$ provides the required pole and its remaining part nicely cancels in the ratio. The product for $l$ not agreeing with $k$ contains two extra terms in the numerator compared to the same term in the denominator, however they cancel by the unitarity of the scattering matrix. The $l=k$ 'th factor in the product exactly reproduces the term required by the kinematical singularity axiom. More details are given in appendix C.

\subsection{The $s u(2)$ sector}

Here we explain the kinematical residue axioms at the tree level for the $s u(2) 3$-pointfunction. The 1-loop calculation is relegated to appendix C. It is convenient to parametrize the momenta by the rapidities, $p(u)$, in terms of which the infinite volume limit of the 3 point function takes the form

$$
C_{123}(\{u\}) \propto=\frac{\prod_{j=1}^{N_{2}} \frac{Q^{(3)}\left(u_{j}^{(2)}\right)}{\left(u_{j}^{(2)}-\frac{i}{2}\right)^{N_{1}}} \prod_{j=1}^{N_{3}}\left(u_{j}^{(3)}-\frac{i}{2}\right)^{N_{1}}}{\prod_{j<k}^{N_{3}}\left(u_{j}^{(3)}-u_{k}^{(3)}+i\right) \prod_{j<k}^{N_{2}}\left(u_{j}^{(2)}-u_{k}^{(2)}+i\right)} D^{[0,1]}
$$

where $D^{[0,1]}$ is given by an $N_{3} \times\left(N_{2}+N_{1}\right)$ determinant

$$
D^{[0,1]}=\left|\begin{array}{ccccccc}
\partial_{u_{1}^{(3)}} T^{(3)}\left(u_{1}^{(2)}\right) & \ldots & \partial_{u_{1}^{(3)}} T^{(3)}\left(u_{N_{2}}^{(2)}\right) & q_{2}\left(u_{1}^{(3)}\right) & \ldots & q_{N_{1}}\left(u_{1}^{(3)}\right) & q_{N_{1}+1}\left(u_{1}^{(3)}\right) \\
\vdots & \vdots & \vdots & \vdots & \vdots & \vdots & \vdots \\
\partial_{u_{N_{3}}^{(3)}} T^{(3)}\left(u_{1}^{(2)}\right) & \ldots & \partial_{u_{N_{3}}^{(3)}} T^{(3)}\left(u_{N_{2}}^{(2)}\right) & q_{2}\left(u_{N_{1}}\right) & \ldots & q_{N_{1}}\left(u_{N_{3}}^{(3)}\right) & q_{N_{1}+1}\left(u_{N_{3}}^{(3)}\right)
\end{array}\right|
$$

and we used Baxter's $Q$-function:

$$
Q^{(i)}(u)=\prod_{j=1}^{N_{i}}\left(u-u_{j}^{(i)}\right) .
$$

The derivative of the transfer matrix can be written in terms of the $s u(2)$ scattering matrix as

$$
\begin{aligned}
\partial_{u_{k}^{(3)}} T^{(3)}\left(u_{j}^{(2)}\right)= & \frac{e^{i p\left(u_{j}^{(2)}\right) L_{3}}}{u_{j}^{(2)}-u_{k}^{(3)}} \frac{i}{u_{j}^{(2)}-u_{k}^{(3)}+i} \frac{Q^{(3)}\left(u_{j}^{(2)}+i\right)}{Q^{(3)}\left(u_{j}^{(2)}\right)} \times \\
& {\left[1-e^{i p\left(u_{j}^{(2)}\right) L_{1}} \prod_{m: m \neq j} S\left(u_{m}^{(2)}, u_{j}^{(2)}\right) \prod_{l: l \neq k} S\left(u_{j}^{(2)}, u_{l}^{(3)}\right)\right] . }
\end{aligned}
$$

In this formula it is legitimate to send $L_{3}$ and $L_{2}$ to infinity and keep $L_{1}=L_{3}-L_{2}$ finite such that the rapidities no longer satisfy the BA equations. As a consequence, 
the expression is singular for $u_{j}^{(2)}=u_{k}^{(3)}$ and we can calculate its residue to check the kinematical singularity axiom. One non-trivial requirement is that the result should be proportional to the 3 -pointfunction with two particles less, when $u_{j}^{(2)}=u_{k}^{(3)}$ were removed. Clearly the overall factors, which depend only on the particles $u_{j}^{(1)}$ factor out. The only singularity at $u_{j}^{(2)}=u_{k}^{(3)}$ comes from the matrix element $\partial_{u_{k}^{(3)}} T^{(3)}\left(u_{j}^{(2)}\right)$. When we expand the determinant w.r.t. this element the subdeterminant is nothing but the determinant, which appears in the reduced 3-point functions and the prefactor is exactly the required one:

$$
\frac{-i \operatorname{Res}_{u_{j}^{(2)}=u_{k}^{(3)} C_{123}(\{u\})}}{C_{123}\left(\{u\} \backslash\left\{u_{j}^{(2)}, u_{k}^{(3)}\right\}\right)} \propto\left[1-e^{i p\left(u_{j}^{(2)}\right) L_{1}} \prod_{m: m \neq j} S\left(u_{m}^{(2)}, u_{j}^{(2)}\right) \prod_{l: l \neq k} S\left(u_{j}^{(2)}, u_{l}^{(3)}\right)\right] .
$$

Finally, let us mention that the $s u(2)$ OPE coefficients exhibit also the bound state pole singularities required by the dynamical singularity axiom.

\section{Conclusions}

In the paper we developed a new framework to determine the (light cone) SFT vertex for integrable worldsheet theories, including as a key special case the $A d S_{5} \times S^{5}$ background.

Our main idea was to use the integrable bootstrap approach to formulate functional relations for the SFT amplitudes incorporating crossing properties and including the scattering matrix of the theory. To achieve this aim we decompactified the worldsheet of the process in which one big string (big J-charge) splits into two smaller ones in two alternative ways. By sending to infinity the sizes of the big string together with any of the other smaller strings allowed to define asymptotic states. The remaining finite string served as a nonlocal operator insertion appearing in the crossing equation in a nontrivial way. The solutions of these functional equations contain exponentially small (wrapping) corrections in the finite string size. After carefully getting rid of these wrapping corrections and applying a straightforward finite size reductions in all of the string sizes we would arrive at a formula valid for any coupling, which incorporates all finite size corrections which are polynomial in the inverse powers of the sizes, but neglects exponentially small corrections. The fact that we can perform this finite size reduction procedure in two different ways, by starting with a finite size for any of the two small strings, gives very strong restrictions for the solutions of the functional relations.

The feasibility of the program was demostrated by reproducing the results for the pp-wave SFT vertex. There, to fix the analytical structure of the solution, we had to assume that the amplitude vanishes for those rapidities of the big string, which were the allowed finite volume states for the undecompactified small string. We do not have a physical explanation for this property but expect similar features for interactive theories. In the asymptotic limit of the SFT vertex we found some effective crossing formulas which bear striking resemblance to the recently discovered modification of crossing in ChernSimons theories.

Although we do not have control of the overall normalization of the SFT vertex, nor do we know the precise general relation between the SFT vertex and OPE coefficients, 
nevertheless we could check some of our diagonal functional equations for the 3-point functions in the weak coupling limit of the AdS/CFT correspondence by comparing them to explicit gauge theory calculations of the OPE coefficients.

We also formulated the axioms for the generic non-diagonal case. For simplicity, and for being in accordance with the rest of the paper, we used rapidity parametrizations $\theta$, with crossing transformations $\theta \rightarrow \theta \pm i \pi$, however, we did not assume any relativistic invariance for the scattering matrix. The generic formulas for the AdS/CFT integrable model can be obtained simply by using its rapidity parametrizations $\theta \rightarrow z$ and together with its crossing transformations $z \rightarrow z \pm \omega_{2}$. These axioms are very similar to the form factor axioms for world-sheet operators except the factor $e^{i p L}$ appearing in the monodromy/periodicity equations and the kinematical singularity axiom. Thus our axioms for the $L=0$ case reduces to them.

An interesting dual line of investigation coming from the weak coupling gauge theory side develops the concept of spin vertex [60-62] (but see also [12]). One qualitative difference between that concept and the functional equations presented in the present paper for the SFT amplitude is that in the worldsheet formulation the wavefunctions of the external states are in a natural way already incorporated into the SFT amplitude (as it deals with multiparticle asymptotic states). On the other hand, the weak coupling approach through the spin vertex concentrates on contractions and loops in the interaction region leaving aside the scalar product with the external states.

There are numerous directions for further research. It would be very important to understand precisely the freedom of choosing a particular solution to the SFT axioms. In particular, even in the pp-wave limit, what are the features of the solution incorporating the correct prefactor. Of course, the solution to the SFT axioms remains an outstanding problem, which is not even solved for the ordinary form factor case i.e. in the $L \rightarrow 0$ limit.

It would be furthermore important to understand the general relation between the SFT vertex and OPE coefficients away from the pp-wave limit (cf. [18, 19, 63]).

Let us emphasize once more that our approach would ultimately provide formulas for the SFT vertex, which are valid for any value of the 't Hooft coupling neglecting wrapping effects. But, even these exponentially small finite size effects are expected to be described in terms of the asymptotic SFT vertex and the scattering matrix of the theory. Their systematic study should also be a direction of future research.

\section{Acknowledgments}

We would like to thank Benjamin Basso, Shota Komatsu, Ivan Kostov, Didina Serban for interesting discussions. We thank Simons Center for Geometry and Physics, Stony Brook for hospitality where a major part of this work was done. RJ was supported by NCN grant 2012/06/A/ST2/00396 and ZB by a Lendület Grant. This work was supported by a Polish-Hungarian Academy of Science cooperation. 


\section{A The decompactified vertex formulation and solution}

The elementary exponential modes for the three strings in the decompactified vertex shown in figure 20 are

$$
\begin{aligned}
\phi_{n}^{(1)}(x) & =\frac{2 \pi}{L} e^{i \frac{2 \pi n x}{L}} & & x \in(-L / 2, L / 2) \\
\phi_{k}^{(2)}(x) & =e^{i k\left(x+\frac{L}{2}\right)} & & x \in(-\infty,-L / 2) \\
& =e^{i k\left(x-\frac{L}{2}\right)} & & x \in(L / 2,+\infty) \\
\phi_{k}^{(3)}(x) & =e^{i k x} & & x \in(-\infty,+\infty) .
\end{aligned}
$$

The 'exponential' overlap matrices are defined through

$$
\tilde{X}_{p k}^{r}=\frac{1}{2 \pi} \int_{-\infty}^{\infty} e^{-i p x} \phi_{k}^{(r)}(x) d x .
$$

For the case at hand, we obtain

$$
\begin{aligned}
\tilde{X}_{p n}^{1} & =\frac{2}{L} \frac{(-1)^{n} \sin \frac{p L}{2}}{p-\frac{2 \pi n}{L}} \\
\tilde{X}_{p k}^{2} & =\cos \frac{p L}{2} \delta(p-k)-\frac{1}{\pi} \sin \frac{p L}{2} P_{\frac{1}{p-k}} \\
\tilde{X}_{p k}^{3} & =\delta(p-k) .
\end{aligned}
$$

The overlap element for string \#2 was obtained using appropriate $e^{\mp \varepsilon x}$ regularization on the two half-lines and the use of

$$
\frac{1}{x \pm i \varepsilon}=\mp i \pi \delta(x)+P_{\frac{1}{x}}
$$

where $P$ stands for the principle value. Since there exist nice factorization properties of the Neumann coefficients when expressed in terms of cosine and sine modes, it is convenient to define (for cosine modes)

$$
X_{p k}^{r} \equiv \frac{1}{2}\left(\tilde{X}_{p k}^{r}+\tilde{X}_{-p k}^{r}+\tilde{X}_{p-k}^{r}+\tilde{X}_{-p-k}^{r}\right)
$$

for positive $p$ and $k$. As reviewed in section 5.1, the Neumann coefficients for negative modes (sine-modes) can be directly obtained from the cosine mode answer. So below we will concentrate exclusively on the positive modes only. Using such modes it has been shown in [59] that the Neumann coefficients can be expressed as

$$
\bar{N}_{n m}^{r s}=-\frac{m n \alpha}{1-4 \mu \alpha K} \frac{\bar{N}_{m}^{r} \bar{N}_{n}^{s}}{\alpha_{s} \omega_{n}^{r}+\alpha_{r} \omega_{m}^{s}} .
$$

In order to provide formulas for $K$ and $\bar{N}_{m}^{r}$ it is customary to introduce quite a lot of notation. We would like to adopt similar notation in the present decompactified case as in the standard finite volume case as used in [59], since we would like to make use of the factorizability proof of [59] which is purely algebraic. To this end let us introduce

$$
C=m \delta_{m n} \quad \text { or } \quad C=p \delta(p-k) .
$$


Figure 20. The decompactified string field theory vertex.

Note that in this appendix the meaning of $\mu$ and $\alpha_{r}$ will be different from the usage in the pp-wave case. However all algebraic relations employed in the factorizability proof will still hold. We will use ${ }^{23}$

$$
\mu \equiv M \quad \alpha_{1}=\frac{L}{2 \pi} \quad \alpha_{2}=1 \quad \alpha_{3}=-1 \quad \alpha=\alpha_{1} \alpha_{2} \alpha_{3}=-\frac{L}{2 \pi}
$$

and

$$
\begin{aligned}
& A_{p k}^{r} \equiv \sqrt{\frac{k}{p}} X_{p k}^{r} \quad C_{r}=\sqrt{C^{2}+\mu^{2} \alpha_{r}^{2}} \\
& U_{(r)}=C^{-1}\left(C_{r}-\mu \alpha_{r} I d\right) \quad U_{(r)}^{-1}=C^{-1}\left(C_{r}+\mu \alpha_{r} I d\right)
\end{aligned}
$$

The key object neccessary for finding the Neuman vectors is the infinite matrix

$$
\Gamma_{+}=\sum_{r=1}^{3} A^{r} U_{(r)} A^{r T}
$$

and an infinite vector $B$ (to be defined below). Then we have

$$
\begin{aligned}
\bar{N}_{m}^{r} & =-\left[\left(C^{-1} C_{r}\right)^{1 / 2} U_{(r)}^{-1} A^{r T} Y\right]_{m} \quad Y \equiv \Gamma_{+}^{-1} B \\
K & =\frac{1}{4} B^{T} \Gamma_{+}^{-1} B
\end{aligned}
$$

The proof of factorizability uses the following properties of the overlap matrices defined above

$$
\begin{array}{rlrl}
A^{r T} C^{-1} A^{s} & =-\frac{\alpha_{r}}{\alpha_{3}} C^{-1} \delta^{r s} & r, s=1,2 \\
\sum_{r=1}^{3} \alpha_{r} A^{r} C^{-1} A^{r T} & =\frac{\alpha}{2} B B^{T} &
\end{array}
$$

\footnotetext{
${ }^{23}$ Note that in this appendix $\alpha_{1}+\alpha_{2}+\alpha_{3} \neq 0$.
} 
The last equation defines for us the vector $B$ (which is related to overlaps with a constant mode). These formulas seem at first glance to be quite convoluted but essentially reflect just the joint completness and mutual orthogonality of modes of strings \#1 and \#2.

The matrices $A_{p k}^{r}$ in the decompactified case are given by

$$
\begin{aligned}
A_{p n}^{1} & =\frac{4}{L} \sqrt{\frac{n}{p}} \frac{(-1)^{n} p \sin \frac{p L}{2}}{p^{2}-\frac{4 \pi^{2} n^{2}}{L^{2}}} \\
A_{p k}^{2} & =\sqrt{\frac{k}{p}}\left(\cos \frac{p L}{2} \delta(p-k)-\frac{1}{\pi} \sin \frac{p L}{2} P_{\frac{2 p}{p^{2}-k^{2}}}\right) \\
A_{p k}^{3} & =\delta(p-k) .
\end{aligned}
$$

One can check that indeed these matrices satisfy (A.16) although this is quite involved and we performed some checks for parts of the formulas only numerically. The equation (A.17) provides for us the expression for the vector $B$ (which in the decompactified case is in fact a function on the positive real line):

$$
B_{p}=\frac{4}{L} p^{-\frac{3}{2}} \sin \frac{p L}{2} .
$$

In the decompactified case that we are considering here, the infinite matrix $\Gamma_{+}$becomes an integral kernel and the vector(function) $Y$ is defined through the integral equation

$$
\int_{0}^{\infty} \Gamma_{+}\left(p, p^{\prime}\right) Y\left(p^{\prime}\right) d p^{\prime}=\frac{4}{L} p^{-\frac{3}{2}} \sin \frac{p L}{2} .
$$

It turns out, however, that even getting an explicit form for the kernel $\Gamma_{+}\left(p, p^{\prime}\right)$ is quite involved.

Using the definition (A.13) and (A.17) we get

$$
\begin{aligned}
\Gamma_{+} & =\sum_{r=1}^{3} A^{r} C^{-1} C_{r} A^{r T}-\frac{\alpha \mu}{2} B B^{T}= \\
& =\sum_{r=1}^{3} A^{r} C^{-1} C_{r} A^{r T}+\frac{4 M}{\pi L}\left(p^{-\frac{3}{2}} \sin \frac{p L}{2}\right)\left(p^{-\frac{3}{2}} \sin \frac{p^{\prime} L}{2}\right) .
\end{aligned}
$$

Let us now give the contributions of the three strings to the above formula:

$$
A^{3} C^{-1} C_{3} A^{3 T}=\frac{1}{p} \sqrt{p^{2}+M^{2}} \delta\left(p-p^{\prime}\right) .
$$

For string \#2 the result is quite messy:

$$
\begin{aligned}
& \int_{0}^{\infty} A_{p k}^{2} \frac{1}{k} \sqrt{k^{2}+M^{2}} A_{p^{\prime} k}^{2} d k= \\
& =\frac{1}{\sqrt{p p^{\prime}}}\left[\sqrt{p^{2}+M^{2}} \delta\left(p-p^{\prime}\right)+\operatorname{Junk}\left(p, p^{\prime}\right)+\frac{4}{\pi^{2}} \sin \frac{p L}{2} \sin \frac{p^{\prime} L}{2} \frac{p F\left(p^{\prime}\right)-p^{\prime} F(p)}{p^{2}-p^{\prime 2}}\right]
\end{aligned}
$$

where

$$
F(p)=\sqrt{p^{2}+M^{2}} \operatorname{arctanh} \frac{p}{\sqrt{p^{2}+M^{2}}}
$$


and

$$
\operatorname{Junk}\left(p, p^{\prime}\right)=-\frac{1}{\pi} \sqrt{p^{2}+M^{2}} \cos \frac{p L}{2} \sin \frac{p^{\prime} L}{2} P_{\frac{2 p^{\prime}}{p^{\prime 2}-p^{2}}}+\left(p \longleftrightarrow p^{\prime}\right) .
$$

The contribution of string \#1 involves a nontrivial infinite sum which can be handled using the techniques of appendix $\mathrm{E}$ in [53].

$$
\begin{aligned}
& \sum_{n=1}^{\infty} A_{p n}^{1} \frac{1}{n} \sqrt{n^{2}+\frac{M^{2} L^{2}}{4 \pi^{2}}} A_{p^{\prime} n}^{1}=\frac{1}{\sqrt{p p^{\prime}}} \frac{16}{L^{2}} \sum_{n=1}^{\infty} \frac{p p^{\prime} \sin \frac{p L}{2} \sin \frac{p^{\prime} L}{2} \sqrt{n^{2}+\frac{M^{2} L^{2}}{4 \pi^{2}}}}{\left(p^{2}-4 n^{2} \frac{\pi^{2}}{L^{2}}\right)\left(p^{\prime 2}-4 n^{2} \frac{\pi^{2}}{L^{2}}\right)} \\
& =\frac{1}{\sqrt{p p^{\prime}}}\left[-\operatorname{Junk}\left(p, p^{\prime}\right)+\frac{1}{\pi^{2}} \sin \frac{p L}{2} \sin \frac{p^{\prime} L}{2} G_{L}\left(p, p^{\prime}\right)-\frac{4 M \sin \frac{p L}{2} \sin \frac{p^{\prime} L}{2}}{\pi L p p^{\prime}}\right]
\end{aligned}
$$

where

$$
G_{L}\left(p, p^{\prime}\right)=-4 p p^{\prime} \int_{M}^{\infty} \frac{\sqrt{\kappa^{2}-M^{2}} \operatorname{coth} \frac{\kappa L}{2} d \kappa}{\left(p^{2}+\kappa^{2}\right)\left(p^{\prime 2}+\kappa^{2}\right)}
$$

When we add all the above ingredients into the formula for $\Gamma_{+}$, we see that the terms which involve $\cos \frac{p L}{2}$ cancel out as well as the $B B^{T}$ term in (A.23). The final formula for $\Gamma_{+}$is thus

$$
\Gamma_{+}\left(p, p^{\prime}\right)=\frac{2}{p} \sqrt{p^{2}+M^{2}} \delta\left(p-p^{\prime}\right)+\frac{1}{\pi^{2} \sqrt{p p^{\prime}}} \sin \frac{p L}{2} \sin \frac{p^{\prime} L}{2}\left[G_{L}\left(p, p^{\prime}\right)+G_{\infty}\left(p, p^{\prime}\right)\right]
$$

and $G_{\infty}\left(p, p^{\prime}\right)$ can be evaluated to be ${ }^{24}$

$$
G_{\infty}\left(p, p^{\prime}\right)=4 \frac{p F\left(p^{\prime}\right)-p^{\prime} F(p)}{p^{2}-p^{\prime 2}} .
$$

The Neumann vectors are now determined by the equation (A.22).

Some comments are in order here. We see here the $\sin \frac{p L}{2}$ factors which eventually make their appearance in the Neumann coefficients even in the infinite $L$ limit. These terms thus arise directly from the decompactified string vertex and are not associated to some subtlety in finite volume reduction. These factors are also quite surprising in that they have a highly oscillatory $L$ behaviour. This leads also to the fact that the large $L$ limit of the equation (A.22) is quite subtle. Indeed we cannot directly take the large $L$ limit of (A.22) but write

$$
\frac{2}{p} \sqrt{p^{2}+M^{2}} Y_{\infty}(p)+\frac{2}{\pi^{2} \sqrt{p}} \sin \frac{p L}{2} \int_{0}^{\infty} G_{\infty}\left(p, p^{\prime}\right) \sin \frac{p^{\prime} L}{2} Y_{\infty}\left(p^{\prime}\right) \frac{d p^{\prime}}{\sqrt{p^{\prime}}}=\frac{4}{L} p^{-\frac{3}{2}} \sin \frac{p L}{2} .
$$

In the large $L$ limit, a consistent solution will have a $\sin \frac{p L}{2}$ factor. After extracting this factor and taking into account that under the integral we may substitute $\sin ^{2} \frac{p^{\prime} L}{2}$ by $\frac{1}{2}$ up to $e^{-M L}$ corrections, we can obtain an integral equation with no explicit $L$ dependence. It is also convenient to pass to rapidity variables. Indeed writing

$$
Y_{\infty}(\theta)=\frac{2}{L M^{\frac{3}{2}}} \sin \frac{p L}{2} \cdot \frac{1}{\sqrt{\sinh \theta} \cosh \theta} h(\theta)
$$

\footnotetext{
${ }^{24}$ It is obtained from $G_{L}\left(p, p^{\prime}\right)$ by replacing the coth by 1 and thus differs by exponential mutliple wrapping terms $e^{-n M L}$.
} 
we get the following integral equation

$$
h(\theta)+\frac{2}{\pi^{2}} \sinh \theta \int_{0}^{\infty} \frac{\theta^{\prime} \sinh \theta \cosh \theta^{\prime}-\theta \cosh \theta \sinh \theta^{\prime}}{\sinh ^{2} \theta-\sinh ^{2} \theta^{\prime}} \frac{h\left(\theta^{\prime}\right) d \theta^{\prime}}{\sinh \theta^{\prime}}=1 .
$$

One can check numerically that this is solved by $h(\theta)=\frac{1}{\sqrt{2}} \sqrt{1+\cosh \theta}$. Thus the large $L$ solution becomes

$$
Y_{\infty}(\theta)=\frac{\sqrt{2}}{L M^{\frac{3}{2}}} \sin \frac{p L}{2} \frac{\sqrt{1+\cosh \theta}}{\cosh \theta \sqrt{\sinh \theta}}
$$

which coincides ${ }^{25}$ with the large $L$ limit of the decompactified LSNS solution $f^{(3)}(\theta)$ following from (4.3) in [52]. Indeed we also verified numerically that its finite $L$ version solves the exact finite $L$ version of the integral equation (A.22). We also checked numerically that the $f^{(2)}(\theta)$ vector is also reproduced. The point of the above exercise was to ascertain that we have full control over the decompactified string vertex solution and make sure that the puzzling $\sin \frac{p L}{2}$ factors indeed arise already for the decompactified vertex and do not come from some complications in subsequent finite volume reduction.

\section{B Properties of the $\tilde{\Gamma}_{\mu}(\theta)$ functions}

In this appendix we summarize the properties of the deformed $\Gamma$ functions. We recall the original definition from [52] and renormalize them in order to simplify the formulas for the Neumann coefficients and to have simpler asymptotic behaviour.

Following [52] we define the $\Gamma_{\mu}(z)$ functions as

$$
\Gamma_{\mu}(z)=\frac{e^{-\gamma \omega_{z}}}{z} \prod_{n=1}^{\infty} \frac{n}{\omega_{n}+\omega_{z}} e^{\frac{\omega_{z}}{n}} ; \quad \omega_{z}=\sqrt{z^{2}+\mu^{2}}
$$

where $\gamma$ is the Euler constant. The authors choose the finite branch cut for the square root $\omega_{-z}=-\omega_{z}$ such that $\Gamma_{\mu}(z)$ satisfies the functional equation:

$$
\Gamma_{\mu}(z) \Gamma_{\mu}(-z)=-\frac{\pi}{z \sin \pi z} .
$$

The large $z$ asymptotic has been calculated to be

$$
\log \Gamma_{\mu}(z)=\omega_{z} \log \frac{\mu}{2 e}+z \log \frac{\omega_{z}+z}{\mu}+\log \frac{\sqrt{2 \pi} \sqrt{\omega_{z}+\mu}}{z}+O\left(e^{-\mu}\right)
$$

or alternatively

$$
\Gamma_{\mu}(z)=\frac{\sqrt{2 \pi} \sqrt{\omega_{z}+\mu}}{z}\left(\frac{\mu}{2 e}\right)^{\omega_{z}}\left(\frac{\omega_{z}+z}{\mu}\right)^{z}+\ldots
$$

The above equations apply only for $|\arg z|<\pi$. For our purposes we renormalize this functions as

$$
\hat{\Gamma}_{\mu}(z)=e^{-\omega_{z} \log \frac{\mu}{2 e}} \Gamma_{\mu}(z) .
$$

\footnotetext{
${ }^{25}$ Upto a $(-1)^{m}$ sign factor.
} 
In order to resolve the branch cut it is convenient do introduce the rapidity parametrization

$$
z=\mu \sinh \theta ; \quad \omega_{z}=\mu \cosh \theta
$$

and consider $\hat{\Gamma}_{\mu}$ as a functions of $\theta$ and $\mu$

$$
\hat{\Gamma}_{\mu}(z) \equiv \tilde{\Gamma}_{\mu}(\theta)
$$

In the $\theta$ variable the two sides of the branch cut are mapped to $\theta$ and $\theta+i \pi$ and the function satisfies

$$
\tilde{\Gamma}_{\mu}(\theta)=-\tilde{\Gamma}_{\mu}(-\theta) ; \quad \tilde{\Gamma}_{\mu}(\theta) \tilde{\Gamma}_{\mu}(\theta+i \pi)=-\frac{\pi}{\mu \sinh \theta \sin (\pi \mu \sinh \theta)} .
$$

Consequently, it is $2 \pi i$ periodic:

$$
\tilde{\Gamma}_{\mu}(\theta+2 \pi i)=\tilde{\Gamma}_{\mu}(\theta)
$$

Moreover, it has the large $\mu$ asymptotical behaviour:

$$
\tilde{\Gamma}_{\mu}(\theta)=\sqrt{\frac{\pi}{\mu}} \frac{e^{\theta \mu \sinh \theta}}{\sinh \frac{\theta}{2}}+\ldots
$$

where $|\Im m(\theta)|<\pi$.

\section{Details on the $s u(1 \mid 1)$ and $s u(2)$ OPE coefficients}

In this appendix we check how the kinematical singularity axiom is satisfied at weak coupling directly for the OPE coefficients. We investigate the 3-point coefficients in the $s u(1 \mid 1)$ and $s u(2)$ sectors up to 1-loop based on the available explicit results obtained from direct gauge theory calculations in $[57,58]$.

As we explained in the main text we have to extract carefully the infinite volume limit of the OPE coefficient from the available finite volume 3-point functions. As we do not know the exact relation between the OPE coeffcients and the SFT vertex we do not bother with the correct normalization of the infinite volume 3-point functions and check the axiom (8.1) only up to proportionality. We start with the simpler $s u(1 \mid 1)$ sector first and proceed with the more complicated $s u(2)$ sector afterwards.

\section{C.1 The $s u(1 \mid 1)$ sector}

In [57] the authors calculated the 3-point function in the $s u(1 \mid 1)$ sector up to 1-loop. They parametrized the operators as closed spin chain Bethe states satisfying the BA equation ${ }^{26}$

$$
e^{i p_{j}^{(r)} L_{r}} \prod_{k: k \neq j}^{N_{r}} S\left(p_{j}^{(r)}, p_{k}^{(r)}\right)=1
$$

\footnotetext{
${ }^{26}$ Here we write the BA equation into our convention. In the AdS convention the BA equation takes usually the form $e^{i p_{j} L}=\prod_{k \neq j} S_{s u(1 \mid 1)}\left(p_{j}, p_{k}\right)$. In [57] $S_{s u(1 \mid 1)}$ was denoted by $S$. Strictly speeking there is also a phase factor difference between $S_{s u(1 \mid 1)}$ and our $S, S_{s u(1 \mid 1)}(p, k)=e^{i(p-k) / 2} S(p, k)^{-1}$, but as it basically shifts the volume we do not keep track of it.
} 
where the scattering matrix in our notation is

$$
S\left(p_{1}, p_{2}\right)=-S_{s u(1 \mid 1)}\left(p_{2}, p_{1}\right)^{-1}=1+8 i g^{2} \sin \frac{p_{1}}{2} \sin \frac{p_{2}}{2} \sin \frac{p_{1}-p_{2}}{2}+O\left(g^{4}\right) .
$$

The OPE coefficients (here we write the complex conjugate of the expression from [57]) up to 1-loop can be written as

$$
C_{123}=\frac{C}{\sqrt{\rho_{N_{1}} \rho_{N_{2}} \rho_{N_{3}}}} \frac{\prod_{r=1}^{3} \prod_{i<j}^{N_{r}} f\left(p_{i}^{(r)}, p_{j}^{(r)}\right)}{\prod_{i}^{N_{3}} \prod_{j}^{N_{2}} f\left(p_{i}^{(3)}, p_{j}^{(2)}\right)} \prod_{k}^{N_{3}}\left(1-e^{-i p_{k}^{(3)} L_{2}} \prod_{i}^{N_{2}} S\left(p_{i}^{(2)}, p_{k}^{(3)}\right)\right)
$$

where

$$
f\left(p_{1}, p_{2}\right)=\left(e^{i p_{1}}-e^{i p_{2}}\right)\left[1-g^{2}\left(1+\cos \left(p_{1}-p_{2}\right)-\cos p_{1}-\cos p_{2}\right)\right]+O\left(g^{4}\right)
$$

and the normalization factor $C$ will not be relevant for our discussion. The original expression in [57] contained factors of the form $\left(1-e^{i p_{k}^{(3)} L_{2}} \prod_{i} S\left(p_{k}^{(3)}, p_{i}^{(2)}\right)\right)$, i.e. the complex conjugate of the expression we wrote. As three point functions seem to be real we could just take the complex conjugate of their result. In any case this does not modify the physics. Let us also note that the appearance of the complex conjugate expression might be related to the conjugate process in which two smaller strings join into a bigger one.

Recalling the general remarks in the main text we can observe that the would be pole of $C_{123}$ at $p_{i}^{(3)}=p_{j}^{(2)}$ is absent due to the zero coming from the BA quantization condition of $p_{j}^{(2)}$. As this OPE coefficient does not have a direct $L_{2} \rightarrow \infty$ limit we use the BA equations for $p_{k}^{(3)}$ to reformulate it following

$$
e^{-i p_{k}^{(3)} L_{2}} \prod_{i}^{N_{2}} S\left(p_{i}^{(2)}, p_{k}^{(3)}\right) \quad \longrightarrow \quad e^{i p_{k}^{(3)} L_{1}} \prod_{i}^{N_{2}} S\left(p_{i}^{(2)}, p_{k}^{(3)}\right) \prod_{j}^{N_{3}} S\left(p_{k}^{(3)}, p_{j}^{(3)}\right) .
$$

We now can safely take the $L_{3}, L_{2} \rightarrow \infty$ limit and obtain the infinite volume 3 -point function

$$
C_{123}(\{p\}) \propto \frac{\prod_{r=1}^{2} \prod_{i<j}^{N_{r}} f\left(p_{i}^{(r)}, p_{j}^{(r)}\right)}{\prod_{i}^{N_{3}} \prod_{j}^{N_{2}} f\left(p_{i}^{(3)}, p_{j}^{(2)}\right)} \prod_{k}^{N_{3}}\left(1-e^{i p_{k}^{(3)} L_{1}} \prod_{i}^{N_{2}} S\left(p_{i}^{(2)}, p_{k}^{(3)}\right) \prod_{j}^{N_{3}} S\left(p_{k}^{(3)}, p_{j}^{(3)}\right)\right)
$$

where the momenta $p^{(3)}$ and $p^{(2)}$ no longer satisfy any quantization condition.

In order to check the kinematical residue axiom we calculate

$$
\frac{-i \operatorname{Res}_{p_{k}^{(3)}=p_{j}^{(2)}} C_{123}(\{p\})}{C_{123}\left(\{p\} \backslash\left\{p_{k}^{(3)}, p_{j}^{(2)}\right\}\right)} .
$$

Using the unitarity of the scattering matrix we obtain the required factor

$$
1-e^{i p_{k}^{(3)} L_{1}} \prod_{i}^{N_{2}} S\left(p_{i}^{(2)}, p_{k}^{(3)}\right) \prod_{j}^{N_{3}} S\left(p_{k}^{(3)}, p_{j}^{(3)}\right)
$$




\section{C.2 The $s u(2)$ sector}

Let us recall the 1-loop structure constant from the literature [58], written in to our conventions when $L_{3}=L_{1}+L_{2}$. The operators are again parametrized by the momenta (more precisely by the spin chain rapidities) of the closed BA states satisfying:

$$
e^{i p\left(u_{k}^{(r)}\right) L_{r}} \prod_{j: j \neq k}^{N_{r}} S\left(u_{k}^{(r)}-u_{j}^{(r)}\right)=1 ; \quad S(u)=S_{s u(2)}(u)=\frac{u-i}{u+i} .
$$

The momenta and the rapidities are related via

$$
e^{i p(u)}=\frac{x\left(u+\frac{i}{2}\right)}{x\left(u-\frac{i}{2}\right)} ; \quad x(u)+\frac{1}{x(u)}=\frac{u}{g} .
$$

The OPE coefficient up to 1-loop takes the form

$$
C_{123}(\{u\})=\frac{C\left(1-g^{2}\left(\Gamma_{1}+\Gamma_{32}^{2}-\alpha_{32}\right)\right)}{\sqrt{\rho_{1} \rho_{2} \rho_{3}}} P_{23} S_{23} A_{1}
$$

where the index refers to the set of rapidities $u_{i}^{(r)}$ the various terms depend on. More explicitly

$$
\Gamma_{i}=\sum_{j=1}^{N_{i}} \frac{1}{\left(u_{j}^{(i)}\right)^{2}+\frac{1}{4}} ; \quad \Gamma_{i j}=\frac{1}{2}\left(\Gamma_{i}-\Gamma_{j}\right)
$$

and

$$
\alpha_{32}=\sum_{j=1}^{N_{i}} \frac{u_{j}^{(3)}}{\left(u_{j}^{(3)}\right)^{2}+\frac{1}{4}}-\sum_{j=1}^{N_{i}} \frac{u_{j}^{(2)}}{\left(u_{j}^{(2)}\right)^{2}+\frac{1}{4}} .
$$

Introducing Baxter's $Q$ functions and the transfer matrices

$$
Q^{(i)}(u)=\prod_{j=1}^{N_{i}}\left(u-u_{j}^{(i)}\right) ; \quad T^{(i)}(u)=\frac{Q^{(i)}(u-i)}{Q^{(i)}(u)}+e^{-i p L_{i}} \frac{Q^{(i)}(u+i)}{Q^{(i)}(u)}
$$

the next term can be written as

$$
P_{23}=\frac{\prod_{j=1}^{N_{2}} \frac{Q^{(3)}\left(u_{j}^{(2)}\right)}{\left(g x\left(u_{j}^{(2)}-\frac{i}{2}\right)\right)^{N_{1}}} \prod_{j=1}^{N_{3}}\left(g x\left(u_{j}^{(3)}-\frac{i}{2}\right)\right)^{N_{1}}}{\prod_{j<k}^{N_{3}}\left(u_{j}^{(3)}-u_{k}^{(3)}+i\right) \prod_{j<k}^{N_{2}\left(u_{j}^{(2)}-u_{k}^{(2)}+i\right)} .}
$$

Finally the most complicated term can be compactly written in terms of a sum of $N_{3} \times$ $\left(N_{1}+N_{2}\right)$ determinants

$$
S_{23}=D^{[0,1]}+g^{2}\left(\left(N_{1}+1\right) D^{[0,3]}+\left(N_{1}-1\right) D^{[1,2]}-2 \alpha_{32} D^{[0,2]}\right)
$$

in which the upper index shows, how the argument of the last two columns are shifted

$$
D^{[i, j]}=\left|\begin{array}{ccccccc}
\partial_{u_{1}^{(3)}} T^{(3)}\left(u_{1}^{(2)}\right) & \ldots & \partial_{u_{1}^{(3)}} T^{(3)}\left(u_{N_{2}}^{(2)}\right) & q_{2}\left(u_{1}^{(3)}\right) & \ldots & q_{N_{1}+i}\left(u_{1}^{(3)}\right) & q_{N_{1}+j}\left(u_{1}^{(3)}\right) \\
\vdots & \vdots & \vdots & \vdots & \vdots & \vdots & \vdots \\
\partial_{u_{N_{3}}^{(3)}} T^{(3)}\left(u_{1}^{(2)}\right) & \ldots & \partial_{u_{N_{3}}^{(3)}} T^{(3)}\left(u_{N_{2}}^{(2)}\right) & q_{2}\left(u_{N_{1}}\right) & \ldots & q_{N_{1}+i}\left(u_{N_{3}}^{(3)}\right) & q_{N_{1}+j}\left(u_{N_{3}}^{(3)}\right)
\end{array}\right|
$$


and

$$
q_{n}(u)=\frac{1}{\left(u+\frac{i}{2}\right)^{n-1}}-\frac{1}{\left(u-\frac{i}{2}\right)^{n-1}} .
$$

The index of $q$ in the $N_{2}+i$ 'th column is $i+1$ except the last two columns which are shifted. The expression for $A_{1}$ is quite involved, however, we do not need its explicit form to check the kinematical singularity axiom.

To prepare for the infinite volume limit we rewrite the transfer matrix in terms of the scattering matrix as

$$
\begin{aligned}
T^{(j)}(u) & =e^{-i p L_{j}} \frac{Q^{(j)}(u+i)}{Q^{(j)}(u)}\left(1+e^{i p L_{j}} \frac{Q^{(j)}(u-i)}{Q^{(j)}(u+i)}\right) \\
& =e^{-i p L_{j}} \frac{Q^{(j)}(u+i)}{Q^{(j)}(u)}\left(1+e^{i p L_{j}} \prod_{k=1}^{N_{j}} S\left(u-u_{k}^{(j)}\right)\right) .
\end{aligned}
$$

The derivative of the transfer matrix appears in the matrix element of the determinant as:

$$
\begin{aligned}
\partial_{u_{k}^{(3)}} T^{(3)}\left(u_{j}^{(2)}\right)= & \frac{e^{-i p\left(u_{j}^{(2)}\right) L_{3}}}{u_{j}^{(2)}-u_{k}^{(3)}} \frac{i}{u_{j}^{(2)}-u_{k}^{(3)}+i} \frac{Q^{(3)}\left(u_{j}^{(2)}+i\right)}{Q^{(3)}\left(u_{j}^{(2)}\right)} \times \\
& {\left[1-e^{i p\left(u_{j}^{(2)}\right) L_{3}} \prod_{k: k \neq j} S\left(u_{j}^{(2)}, u_{k}^{(3)}\right)\right] . }
\end{aligned}
$$

Clearly the $u_{j}^{(2)}=u_{k}^{(3)}$ would be pole is annihilated by the zero which manifests the BA equation. In order to have a well defined $L_{3}, L_{2} \rightarrow \infty$ limit we use the BA of $u_{j}^{(2)}$ to rewrite this expression as

$$
\begin{aligned}
\partial_{u_{k}(3)} T^{(3)}\left(u_{j}^{(2)}\right)= & \frac{e^{-i p\left(u_{j}^{(2)}\right) L_{3}}}{u_{j}^{(2)}-u_{k}^{(3)}} \frac{i}{u_{j}^{(2)}-u_{k}^{(3)}+i} \frac{Q^{(3)}\left(u_{j}^{(2)}+i\right)}{Q^{(3)}\left(u_{j}^{(2)}\right)} \times \\
& {\left[1-e^{i p\left(u_{j}^{(2)}\right) L_{3}} \prod_{m: m \neq j} S\left(u_{m}^{(2)}, u_{j}^{(2)}\right) \prod_{k: k \neq j} S\left(u_{j}^{(2)}, u_{k}^{(3)}\right)\right] . }
\end{aligned}
$$

Now it is legitimate to send $L_{3}$ and $L_{2}$ to infinity and keep $L_{1}=L_{3}-L_{2}$ finite. This procedure, together with the renormalization of the BA states to the infinite volume scattering basis, result in the infinite volume 'OPE coefficient', where the rapidities no longer satisfy the BA equation, i.e. they are off-shell. As a consequence, the expression is singular for $u_{j}^{(2)}=u_{k}^{(3)}$ and we can calculate its residue to check the kinematical singularity axiom. One non-trivial requirement is that the result should be proportional to a similar decompactified OPE coefficient with two particles less, when $u_{j}^{(2)}=u_{k}^{(3)}$ were removed. Clearly the overall factors, which depend only on the particles $u_{j}^{(1)}$ factor out. Additionally $\Gamma_{32}$ and $\alpha_{32}$ reduces to the analogous expression with two particles less. The only singularity at $u_{j}^{(2)}=u_{k}^{(3)}$ comes from the common matrix element $\partial_{u_{k}^{(3)}} T^{(3)}\left(u_{j}^{(2)}\right)$ of all determinants. When we expand the determinants w.r.t. this element the subdeterminant is nothing but 
the determinant, which appears in the reduced OPE coefficient and the prefactor is exactly the required one:

$$
\frac{-i \operatorname{Res}_{u_{j}^{(2)}=u_{k}^{(3)} C_{123}(\{u\})}}{C_{123}\left(\{u\} \backslash\left\{u_{j}^{(2)}, u_{k}^{(3)}\right\}\right)} \propto\left[1-e^{i p\left(u_{j}^{(2)}\right) L_{1}} \prod_{m: m \neq j} S\left(u_{m}^{(2)}, u_{j}^{(2)}\right) \prod_{l: l \neq k} S\left(u_{j}^{(2)}, u_{l}^{(3)}\right)\right] .
$$

Open Access. This article is distributed under the terms of the Creative Commons Attribution License (CC-BY 4.0), which permits any use, distribution and reproduction in any medium, provided the original author(s) and source are credited.

\section{References}

[1] N. Beisert et al., Review of AdS/CFT Integrability: An Overview, Lett. Math. Phys. 99 (2012) 3 [arXiv: 1012.3982] [INSPIRE].

[2] J.M. Maldacena, The large- $N$ limit of superconformal field theories and supergravity, Int. J. Theor. Phys. 38 (1999) 1113 [hep-th/9711200] [INSPIRE].

[3] G. Arutyunov and S. Frolov, String hypothesis for the $A d S_{5} \times S^{5}$ mirror, JHEP 03 (2009) 152 [arXiv:0901.1417] [INSPIRE].

[4] N. Gromov, V. Kazakov and P. Vieira, Exact Spectrum of Anomalous Dimensions of Planar $N=4$ Supersymmetric Yang-Mills Theory, Phys. Rev. Lett. 103 (2009) 131601 [arXiv: 0901.3753] [INSPIRE].

[5] D. Bombardelli, D. Fioravanti and R. Tateo, Thermodynamic Bethe Ansatz for planar AdS/CFT: A Proposal, J. Phys. A 42 (2009) 375401 [arXiv:0902.3930] [InSPIRE].

[6] J. Balog and A. Hegedus, Hybrid-NLIE for the AdS/CFT spectral problem, JHEP 08 (2012) 022 [arXiv: 1202.3244] [INSPIRE].

[7] N. Gromov, V. Kazakov, S. Leurent and D. Volin, Quantum Spectral Curve for Planar $\mathcal{N}=4$ super-Yang-Mills Theory, Phys. Rev. Lett. 112 (2014) 011602 [arXiv:1305.1939] [INSPIRE].

[8] N. Gromov, V. Kazakov, S. Leurent and D. Volin, Quantum spectral curve for arbitrary state/operator in $A d S_{5} / \mathrm{CFT}_{4}$, arXiv: 1405.4857 [INSPIRE].

[9] N.R. Constable et al., PP wave string interactions from perturbative Yang-Mills theory, JHEP 07 (2002) 017 [hep-th/0205089] [INSPIRE].

[10] N. Beisert, C. Kristjansen, J. Plefka, G.W. Semenoff and M. Staudacher, BMN correlators and operator mixing in $N=4$ super Yang-Mills theory, Nucl. Phys. B 650 (2003) 125 [hep-th/0208178] [INSPIRE].

[11] L.F. Alday, J.R. David, E. Gava and K.S. Narain, Structure constants of planar $N=4$ Yang-Mills at one loop, JHEP 09 (2005) 070 [hep-th/0502186] [INSPIRE].

[12] L.F. Alday, J.R. David, E. Gava and K.S. Narain, Towards a string bit formulation of $N=4$ super Yang-Mills, JHEP 04 (2006) 014 [hep-th/0510264] [INSPIRE].

[13] D.E. Berenstein, J.M. Maldacena and H.S. Nastase, Strings in flat space and pp waves from $N=4$ super Yang-Mills, JHEP 04 (2002) 013 [hep-th/0202021] [INSPIRE].

[14] M. Spradlin and A. Volovich, Superstring interactions in a pp wave background, Phys. Rev. D 66 (2002) 086004 [hep-th/0204146] [INSPIRE].

[15] A. Pankiewicz and B. Stefański Jr., PP wave light cone superstring field theory, Nucl. Phys. B 657 (2003) 79 [hep-th/0210246] [INSPIRE]. 
[16] C.-S. Chu and V.V. Khoze, Correspondence between the three point BMN correlators and the three string vertex on the pp wave, JHEP 04 (2003) 014 [hep-th/0301036] [INSPIRE].

[17] P. Di Vecchia, J.L. Petersen, M. Petrini, R. Russo and A. Tanzini, The three string vertex and the AdS/CFT duality in the PP wave limit, Class. Quant. Grav. 21 (2004) 2221 [hep-th/0304025] [INSPIRE].

[18] S. Dobashi and T. Yoneya, Resolving the holography in the plane-wave limit of AdS/CFT correspondence, Nucl. Phys. B 711 (2005) 3 [hep-th/0406225] [INSPIRE].

[19] H. Shimada, Holography at string field theory level: Conformal three point functions of BMN operators, Phys. Lett. B 647 (2007) 211 [hep-th/0410049] [INSPIRE].

[20] R.R. Metsaev and A.A. Tseytlin, Exactly solvable model of superstring in Ramond-Ramond plane wave background, Phys. Rev. D 65 (2002) 126004 [hep-th/0202109] [INSPIRE].

[21] M.B. Green, J.H. Schwarz and L. Brink, Superfield Theory of Type II Superstrings, Nucl. Phys. B 219 (1983) 437 [INSPIRE].

[22] A.B. Zamolodchikov and A.B. Zamolodchikov, Factorized s Matrices in Two-Dimensions as the Exact Solutions of Certain Relativistic Quantum Field Models, Annals Phys. 120 (1979) 253 [INSPIRE].

[23] G. Mussardo, Off critical statistical models: Factorized scattering theories and bootstrap program, Phys. Rept. 218 (1992) 215 [INSPIRE].

[24] P. Dorey, Exact $S$ matrices, hep-th/9810026 [INSPIRE].

[25] A.B. Zamolodchikov, Thermodynamic Bethe Ansatz in Relativistic Models. Scaling Three State Potts and Lee-yang Models, Nucl. Phys. B 342 (1990) 695 [InSPIRE].

[26] P. Dorey and R. Tateo, Excited states by analytic continuation of TBA equations, Nucl. Phys. B 482 (1996) 639 [hep-th/9607167] [InSPIRE].

[27] S. Ghoshal and A.B. Zamolodchikov, Boundary $S$ matrix and boundary state in two-dimensional integrable quantum field theory, Int. J. Mod. Phys. A 9 (1994) 3841 [Erratum ibid. A 9 (1994) 4353] [hep-th/9306002] [INSPIRE].

[28] G. Delfino, G. Mussardo and P. Simonetti, Statistical models with a line of defect, Phys. Lett. B 328 (1994) 123 [hep-th/9403049] [INSPIRE].

[29] M. Karowski and P. Weisz, Exact Form-Factors in (1+1)-Dimensional Field Theoretic Models with Soliton Behavior, Nucl. Phys. B 139 (1978) 455 [InSPIRE].

[30] F.A. Smirnov, Form-factors in completely integrable models of quantum field theory, Adv. Ser. Math. Phys. 14 (1992) 1.

[31] H.M. Babujian, A. Fring, M. Karowski and A. Zapletal, Exact form-factors in integrable quantum field theories: The sine-Gordon model, Nucl. Phys. B 538 (1999) 535 [hep-th/9805185] [INSPIRE].

[32] L.F. Alday and J.M. Maldacena, Gluon scattering amplitudes at strong coupling, JHEP 06 (2007) 064 [arXiv:0705.0303] [INSPIRE].

[33] R.A. Janik and A. Wereszczynski, Correlation functions of three heavy operators: The AdS contribution, JHEP 12 (2011) 095 [arXiv:1109.6262] [INSPIRE].

[34] Y. Kazama and S. Komatsu, On holographic three point functions for GKP strings from integrability, JHEP 01 (2012) 110 [Erratum ibid. 1206 (2012) 150] [arXiv:1110.3949] [INSPIRE].

[35] Y. Kazama and S. Komatsu, Wave functions and correlation functions for GKP strings from integrability, JHEP 09 (2012) 022 [arXiv: 1205.6060] [INSPIRE]. 
[36] Y. Kazama and S. Komatsu, Three-point functions in the $\mathrm{SU}(2)$ sector at strong coupling, JHEP 03 (2014) 052 [arXiv: 1312.3727] [INSPIRE].

[37] B. Basso, A. Sever and P. Vieira, Spacetime and Flux Tube S-Matrices at Finite Coupling for $N=4$ Supersymmetric Yang-Mills Theory, Phys. Rev. Lett. 111 (2013) 091602 [arXiv: 1303.1396] [INSPIRE].

[38] M. Lüscher, Volume Dependence of the Energy Spectrum in Massive Quantum Field Theories. 1. Stable Particle States, Commun. Math. Phys. 104 (1986) 177 [inSPIRE].

[39] Z. Bajnok and R.A. Janik, Four-loop perturbative Konishi from strings and finite size effects for multiparticle states, Nucl. Phys. B 807 (2009) 625 [arXiv:0807.0399] [INSPIRE].

[40] D. Bombardelli, A next-to-leading Lüscher formula, JHEP 01 (2014) 037 [arXiv:1309.4083] [INSPIRE].

[41] Z. Bajnok, The formfactor bootstrap, talk at Nordita Workshop: Exact Results in Gauge-String Dualities, Nordita, Stockholm, 6-10 February 2012.

[42] T. Klose and T. McLoughlin, Worldsheet Form Factors in AdS/CFT, Phys. Rev. D 87 (2013) 026004 [arXiv: 1208.2020] [INSPIRE].

[43] T. Klose and T. McLoughlin, Comments on World-Sheet Form Factors in AdS/CFT, J. Phys. A 47 (2014) 055401 [arXiv: 1307.3506] [INSPIRE].

[44] Z. Bajnok, R.A. Janik and A. Wereszczyński, HHL correlators, orbit averaging and form factors, JHEP 09 (2014) 050 [arXiv:1404.4556] [INSPIRE].

[45] A.B. Zamolodchikov, Two point correlation function in scaling Lee-Yang model, Nucl. Phys. B 348 (1991) 619 [INSPIRE].

[46] A. Fring, G. Mussardo and P. Simonetti, Form-factors for integrable Lagrangian field theories, the sinh-Gordon theory, Nucl. Phys. B 393 (1993) 413 [hep-th/9211053] [InSPIRE].

[47] B. Pozsgay and G. Takács, Form-factors in finite volume I: Form-factor bootstrap and truncated conformal space, Nucl. Phys. B 788 (2008) 167 [arXiv:0706.1445] [INSPIRE].

[48] K. Zarembo, Holographic three-point functions of semiclassical states, JHEP 09 (2010) 030 [arXiv: 1008.1059] [INSPIRE].

[49] M.S. Costa, R. Monteiro, J.E. Santos and D. Zoakos, On three-point correlation functions in the gauge/gravity duality, JHEP 11 (2010) 141 [arXiv:1008.1070] [INSPIRE].

[50] Z. Bajnok and R.A. Janik, HHL correlators and diagonal form factors, to appear.

[51] W. Schulgin and A.V. Zayakin, Three-BMN Correlation Functions: Integrability vs. String Field Theory. One-Loop Mismatch, JHEP 10 (2013) 053 [arXiv: 1305.3198] [InSPIRE].

[52] J. Lucietti, S. Schäfer-Nameki and A. Sinha, On the plane wave cubic vertex, Phys. Rev. D 70 (2004) 026005 [hep-th/0402185] [INSPIRE].

[53] Y.-H. He, J.H. Schwarz, M. Spradlin and A. Volovich, Explicit formulas for Neumann coefficients in the plane wave geometry, Phys. Rev. D 67 (2003) 086005 [hep-th/0211198] [INSPIRE].

[54] S. Jain, M. Mandlik, S. Minwalla, T. Takimi, S.R. Wadia and S. Yokoyama, Unitarity, Crossing Symmetry and Duality of the S-matrix in large-N Chern-Simons theories with fundamental matter, arXiv:1404.6373 [INSPIRE].

[55] Y. Dandekar, M. Mandlik and S. Minwalla, Poles in the S-Matrix of Relativistic Chern-Simons Matter theories from Quantum Mechanics, arXiv:1407.1322 [INSPIRE].

[56] V.P. Yurov and A.B. Zamolodchikov, Correlation functions of integrable $2-D$ models of relativistic field theory. Ising model, Int. J. Mod. Phys. A 6 (1991) 3419 [InSPIRE]. 
[57] J. Caetano and T. Fleury, Three-point functions and $\mathfrak{s u}(1 \mid 1)$ spin chains, JHEP 09 (2014) 173 [arXiv: 1404.4128] [INSPIRE].

[58] N. Gromov and P. Vieira, Tailoring Three-Point Functions and Integrability IV. Theta-morphism, JHEP 04 (2014) 068 [arXiv:1205.5288] [INSPIRE].

[59] J.H. Schwarz, Comments on superstring interactions in a plane wave background, JHEP 09 (2002) 058 [hep-th/0208179] [INSPIRE].

[60] Y. Kazama, S. Komatsu and T. Nishimura, Novel construction and the monodromy relation for three-point functions at weak coupling, JHEP 01 (2015) 095 [arXiv:1410.8533] [INSPIRE].

[61] Y. Jiang, I. Kostov, A. Petrovskii and D. Serban, String Bits and the Spin Vertex, arXiv: 1410.8860 [INSPIRE].

[62] Y. Jiang and A. Petrovskii, From Spin Vertex to String Vertex, arXiv:1412.2256 [INSPIRE].

[63] T. Klose and T. McLoughlin, A light-cone approach to three-point functions in $A d S_{5} \times S^{5}$, JHEP 04 (2012) 080 [arXiv:1106.0495] [INSPIRE]. 